\title{
Assessment of the apparent bending stiffness and damping of multilayer plates; modelling and experiment
}

\author{
Kerem Ege* \\ Univ Lyon, INSA-Lyon, Laboratoire Vibrations Acoustique, LVA EA677, \\ F-69621 Villeurbanne, France
}

N.B. Roozen

KU Leuven, Laboratory of Acoustics, Soft matter and Biophysics, Department of Physics and Astronomy, Celestijnenlaan 200D, B-3001 Heverlee, Belgium

Quentin Leclère

Univ Lyon, INSA-Lyon, Laboratoire Vibrations Acoustique, LVA EA677, F-69621 Villeurbanne, France

Renaud G. Rinaldi

MATEIS CNRS UMR5510, INSA-Lyon, 7 Avenue Jean Capelle, F-69621 Villeurbanne Cedex, France

\begin{abstract}
In the context of aeronautics, automotive and construction applications, the design of light multilayer plates with optimized vibroacoustical damping and isolation performances remains a major industrial challenge and a hot topic of research. This paper focuses on the vibrational behavior of three-layered sandwich composite plates in a broad-band frequency range. Several aspects are studied through measurement techniques and analytical modelling of a steel/polymer/steel plate sandwich system. A contactless measurement of the velocity field of plates using a scanning laser vibrometer is performed, from which the equivalent single layer complex rigidity (apparent bending stiffness and apparent damping) in the mid/high frequency ranges is estimated. The results are combined with low/mid frequency estimations obtained with a high-resolution modal analysis method so that the frequency dependent equivalent Young's modulus and equivalent loss factor of the composite plate are identified for the whole $[40 \mathrm{~Hz}-20 \mathrm{kHz}]$ frequency band. The results are in very good agreement with an equivalent single layer analytical modelling based on wave propagation analysis (model of Guyader). The comparison with this model allows identifying the frequency dependent complex modulus of the polymer core layer through inverse
\end{abstract}


resolution. Dynamical mechanical analysis measurements are also performed on the polymer layer alone and compared with the values obtained through the inverse method. Again, a good agreement between these two estimations over the broad-band frequency range demonstrates the validity of the approach.

Key words: Determination of viscoelastic material properties; Prediction/measurement comparisons; Hybrid sandwich panels; Equivalent plate model; Apparent bending stiffness and apparent loss factor; DMA/vibratory comparisons

\section{Introduction}

The combined high stiffness and light weight of sandwich composites make them increasingly used by today's transportation and construction industries for example. In this context, the design of light multilayer plates with optimized damping and isolation performances, for given frequency bands, remains a major industrial challenge and a hot topic of research. Thus, this article concerns the vibrational behavior of such lightweight composite plates in a broad-band frequency range.

Three analytical and four experimental vibroacoustics methods identifying the equivalent complex bending stiffness (or flexural rigidity) and equivalent loss factor of a three-layered plate are compared. These equivalent parameters, also known in the literature as apparent stiffness and apparent loss factor (see for example the studies from Nilsson [1] or Backström [2]), are of course frequency dependent. The purpose of this work is then to identify them up to the high-frequency domain where most of the experimental methods meet their limits in terms of precision and resolution (frequency or spatially). Several techniques are reported in the literature to handle such limitations, in particular wave number fitting approaches (see for example the work of Berthaut et al. [3] on ribbed structures and the one of Cherif et al. [4] on composite panels with honey comb core), image source methodologies (see for example the method of Cuenca et al. for estimating material properties of plates $[5,6])$ or the recently proposed Virtual Fields Method combined with Laser Doppler Vibrometry or optical deflectometry by Berry et. al. [7,8]. Here we made the choice to compare four experimental approaches: traditional modal analysis [9], high-resolution modal analysis [10], CFAT methodology that uses a corrected finite differences scheme [11], and a wave correlation technique comprising an image source model that uses Hankel's functions [12].

\footnotetext{
* corresponding author

Email addresses: kerem.ege@ insa-lyon. fr (Kerem Ege),

bert.roozen@kuleuven.be (N.B. Roozen),

quentin.lecleredinsa-lyon.fr(Quentin Leclère),

renaud.rinaldi@insa-lyon.fr(Renaud G. Rinaldi).
} 
Concerning the comparison with predictions, different approaches exist to model the vibrational behavior of multilayer plates (see for example a synthesis done by Carrera [13] or more recently the work of Shorter [14], Manconi and Mace [15], and Ghinet and Atalla [16], for thick composite laminated panels). Here three models are presented, compared and discussed in the first part of the manuscript - a) model of Guyader (traveling wave approach) $[17,18]$ b) model of Ross, Kerwin and Ungar (strain energy) $[19,20,21]$ c) Lamb waves model [22]. The plate under study (a steel/polymer/steel sandwich system) is then presented, and the experimental protocols and assessment procedures of the four experimental techniques considered are detailed in section 3. Using traditional modal analysis in the low frequency domain and finite-element model (FEM) calculations, the frequency dependent equivalent Young's modulus (derived from the equivalent bending stiffness) is identified up to $800 \mathrm{~Hz}$. Then, a high-resolution modal analysis method allows identifying equivalent loss factor as function of frequency up to $2.5-3 \mathrm{kHz}$ where modal overlap is high. Using two different methodologies (CFAT methodology and Hankel's functions image source model), contactless measurements of the velocity field extend the identification of the frequency dependent complex Young's modulus up to the very high frequency domain $(20 \mathrm{kHz})$ and confirm both previous modal analyses estimations. Measurements and predictions are then compared and discussed in section 4 . The article ends with the identification of the polymer core complex modulus that is finally compared with DMA measurements/extrapolations.

The chosen analytical and experimental methods do not limit this work to a benchmark study but rather aims at the discussion of measurement approaches that cover a wide frequency range. These measurements are validated with predictions, focusing on damping identification which is not straightforward in cases of high modal overlaps. Hence - extending a previous oral contribution [23] where only preliminary experimental results were given without theoretical discussions and fair comparisons - the principal novelties of this work can be summarized as follows:

- comparing for a given three-layered plate experimental modal analyses techniques (in the low/mid-frequency domains) to wave number fitting approaches (up to very high frequencies) with a focus on accurate damping characterisation

- obtaining - over the whole [40 Hz-20 kHz] frequency range - overlapping experimental results in good agreement with an equivalent single layer plate model with frequency dependent apparent bending stiffness and damping

- using modelling predictions to identify through an inverse vibratory problem the missing mechanical properties of a given layer (here the polymeric core complex Young's modulus) and confirming this results with DMA measurements/extrapolations. 


\section{Equivalent single layer modelling}

In this section three analytical modelling of multilayer are presented, compared and discussed on two examples of sandwich plates. Only main principles of the methods are recalled in this first section; more details on each of the techniques are given in appendices and corresponding cited references.

\subsection{Model of Guyader}

The first analytical approach considered is the model developed by Guyader et al. $[17,18]$ which determines at a given frequency the equivalent complex flexural rigidity (or bending stiffness) $D_{\mathrm{eq}}^{*}(f)=D_{\mathrm{eq}}(f)\left(1+\mathrm{j} \eta_{\mathrm{eq}}(f)\right)$ of a multilayer viscoelastic plate in the standard Love-Kirchhoff thin plate theory. The analytical method is based on the travelling wave approach applied to a simplified multilayer model. The bending, membrane and shear effects of each layers are considered and continuity conditions on displacement and shear stresses at each layer interface are used to obtain the equation of motion of the multilayer plate field expressed as a function of the first layer field. Hence the method determines for a given frequency of calculation the equivalent single layer plate complex bending stiffness under Love-Kirchhoff thin plate theory in order to exhibit the same transverse displacement that the multilayer plate. Details on the analytical derivation of this first approach considered here can be found in $[17,18]$. Note that some typing errors in the appendix of [17] have been corrected in the reimplementation of the model that was used in this paper.

Once this equivalent rigidity $D_{\text {eq }}^{*}(f)$ at a given frequency $f$ has been computed, the equivalent homogeneous material properties - density $\rho_{\mathrm{eq}}$, Poisson's ratio $\nu_{\mathrm{eq}}$, Young's modulus $E_{\mathrm{eq}}(f)$ and loss factor $\eta_{\mathrm{eq}}(f)$ - of the multilayer are obtained as follow:

$$
\begin{aligned}
\rho_{\mathrm{eq}} & =\frac{\sum_{i} h_{i} \rho_{i}}{\sum_{i} h_{i}} \\
\nu_{\mathrm{eq}} & =\frac{\sum_{i} h_{i} \nu_{i}}{\sum_{i} h_{i}} \\
E_{\mathrm{eq}}(f) & =\Re\left(D_{\mathrm{eq}}^{*}(f)\right) \frac{12\left(1-\nu_{\mathrm{eq}}^{2}\right)}{h^{3}} \\
\eta_{\mathrm{eq}}(f) & =\frac{\Im\left(D_{\mathrm{eq}}^{*}(f)\right)}{\Re\left(D_{\mathrm{eq}}^{*}(f)\right)}
\end{aligned}
$$

where $h=\sum h_{i}$ is the total thickness of the composite panel and the subscripts "eq" and " $"$ refer to the equivalent homogenized parameters of the multilayer and the $\mathrm{i}^{\text {th }}$ layer, respectively. Naturally the equivalent homogeneous Young's modulus and 
the equivalent homogeneous loss factor depend on the frequency $f$.

Ultimately, the only parameters needed for the equivalent single layer modelling are thicknesses $h_{i}$, densities $\rho_{i}$ and viscoelastic characteristics (Young's modulus $E_{i}$, loss factor $\eta_{i}$, Poisson's ratio $\nu_{i}$ ) of each layer. For the steel/polymer/steel plate that will be studied in section 3 and 4, the material properties for steel as known from the literature were used. The density of the polymer layer was measured, while the Young's modulus and loss factor of this layer was fitted on the measurement data (see paragraph 4.1).

\subsection{Model of Ross, Kerwin and Ungar}

The second model considered make several simplifying assumptions compared to Guyader's approach. The model was originally due to Ross, Kerwin and Ungar [19], which was accompanied by publications of Kerwin [20] and Ungar [21]. This model will be denoted here by the $R K U$ model (as commonly done in the literature, in [24] for example). The framework considers the dynamics of a constrained layer system being composed of three layers ; a simplified energetic approach (based on the dissipation of power through shear motion of the damping layer) is developed to estimate the equivalent complex flexural rigidity of the structure. Under several assumptions (thin and soft interlayer thus effectively only accounting for shear; no shear deformation of the skin or membrane effects) this simplified model relates the shear strain of the damping layer to the transverse motion (bending waves) of the three-layer. The theory of Ross, Kerwin and Ungar is briefly outlined in Appendix A.

\subsection{Lamb waves model}

In the field of ultrasonic non-destructive testing, the Lamb waves are extensively used (see for instance [25], [26]). The modelling of Lamb waves is a classical topic, which was addressed in the standard text by Viktorov [22], dating back to the seventies of the previous century. Lamb waves model is exact in that no assumption are made with respect to the frequency range in which it is valid. In that sense the Lamb waves model differs from models that use a low frequency approximation of bending waves (it is worth pointing out that this approximation applies to both Guyader and RKU model). This exactness of Lamb waves model is relevant for the present study, since it allows the verification of the validity of the two previous models up to high frequencies. The theory of Viktorov, in which each layer is modeled individually by means of a Lamb waves model, is briefly outlined in Appendix B. 


\subsection{Analytical comparison and discussion}

Two hybrid sandwich composite panels, often depicted as constrained-layer damping sandwich plates in the literature, are considered in this section for analytical comparison. Both are constituted of three homogeneous isotropic layers (two metal face sheets and a polymer core):

- one three-layered system with steel skin layers and with a thin and soft flexural polymer interlayer (hypotheses of RKU model) called SOFT plate

- one steel/polymer/steel plate with a stiffer polymer interlayer called SPS plate

The input parameters of each layers of the plates are summarized in Table 1.

A sample of the SPS plate will be experimentally studied in section 3. Standard values for the material properties of the steel skin layers have been selected. The thicknesses of the layers have been measured and the density of the polymer core determined from weight and dimensions measurements. The complex Young's modulus of the polymer core has been chosen here in agreement with the vibratory identifications and DMA measurements (presented in the last part of the manuscript). Concerning the values taken for the SOFT plate for this analytical comparison part, they have been chosen so that the assumptions made on the RKU model are being met that is [20], [21]:

- the Young's modulus of layer 2 is taken to be small relative to that of layers 1 and 3

- the bending stiffness of layer 3 is small with respect to the bending stiffness of layer 1: $E_{3} h_{3}^{3} \ll E_{1} h_{1}^{3}$

To this end we chose here for this SOFT plate a Young's modulus of the core ten times softer than for SPS case.

The predictions of the three models are compared in figure 1 and figure 2 for the SOFT and SPS plates respectively; relevant analytical results are also gathered in table 2. Asymptotic values at low and high frequencies have been added to the figures (horizontal dashed lines) using equation A.9 and A.10 for the equivalent rigidity and A.11 and A.12 for the equivalent loss factor. The frequency for which the optimum shearing of the dissipative core layers occurs (shear parameter equal to $X_{\mathrm{opt}}$ in the RKU model (see equation A.13) is also added to the figures (vertical red dashed line), together with the maximum damping factor frequency in the model of Guyader (vertical blue dashed line).

The frequency evolution of the equivalent rigidity and loss factor is typical for such constrained-layer damping sandwich plates (see for example the studies reported by Nilsson [1] or Backström [2]), that is: 


\begin{tabular}{|c||c|c|c|}
\hline SOFT plate & layer 1 (steel) & layer 2 (polymer) & layer 3 (steel) \\
\hline Thickness $h[\mathrm{~m}]$ & $0.8 \times 10^{-3}$ & $0.4 \times 10^{-3}$ & $0.1 \times 10^{-3}$ \\
Young's modulus $E[\mathrm{GPa}]$ & 210 & 0.03 & 210 \\
Density $\rho\left[\mathrm{kg} \cdot \mathrm{m}^{-3}\right]$ & 7800 & 580 & 7800 \\
Poisson's ratio $\nu[-]$ & 0.33 & 0.33 & 0.33 \\
Loss factor $\eta[-]$ & - & 0.06 & - \\
\hline SPS plate & layer 1 (steel) & layer 2 (polymer) & layer 3 (steel) \\
\hline Thickness $h[\mathrm{~m}]$ & $0.18 \times 10^{-3}$ & $0.69 \times 10^{-3}$ & $0.18 \times 10^{-3}$ \\
Young's modulus $E[\mathrm{GPa}]$ & 210 & 0.3 & 210 \\
Density $\rho\left[\mathrm{kg} . \mathrm{m}^{-3}\right]$ & 7800 & 580 & 7800 \\
Poisson's ratio $\nu[-]$ & 0.33 & 0.33 & 0.33 \\
Loss factor $\eta[-]$ & 0.005 & 0.03 & 0.005 \\
\hline
\end{tabular}

Table 1

Dimensions and material properties of the individual layers for the two constrained-layer damping sandwich plates chosen for analytical comparison.
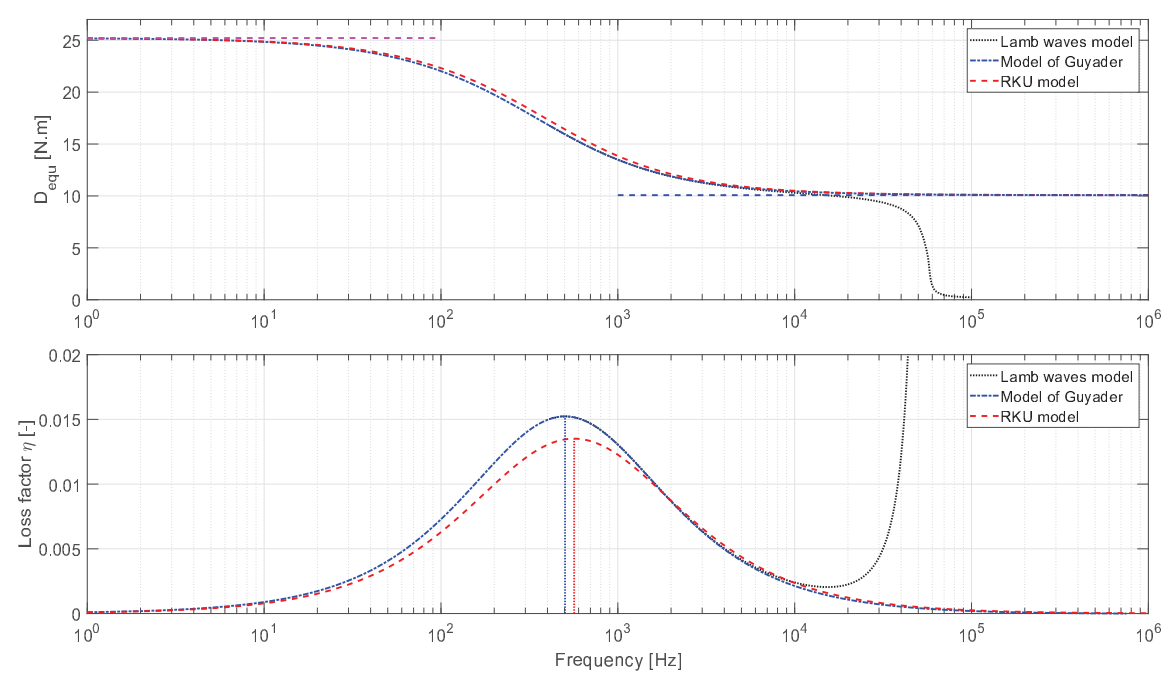

Figure 1. Comparison of the three models for the thin and soft flexural polymer interlayer "SOFT" plate - Equivalent rigidity $D_{\text {eq }}(f)$ (top) ; Equivalent loss factor $\eta_{\text {eq }}$ (bottom). Lowand high-frequency asymptotic values are added in horizontal dashed lines, and maximum damping frequency is added in vertical dashed lines for both RKU model and Guyader's model.

- for the equivalent rigidity: a constant value (high rigidity) at low frequency decreasing gradually to a lower asymptotic value at very high frequencies.

- for the equivalent loss factor: small damping at low- and very high-frequencies (close to the metallic skin loss factor value), and high damping for a limited 

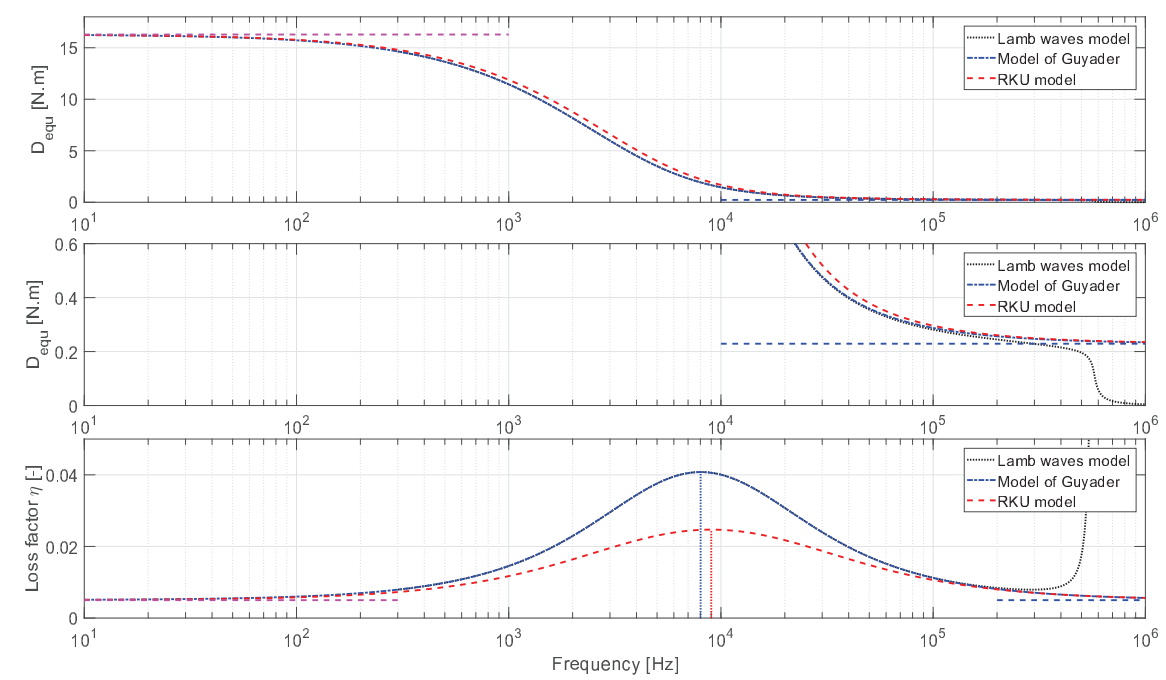

Figure 2. Comparison of the three models for the steel-polymer-steel "SPS" plate - Equivalent rigidity $D_{\mathrm{eq}}(f)$ at top and middle (zoom in high-frequency) ; Equivalent loss factor $\eta_{\mathrm{eq}}$ at bottom. Low- and high-frequency asymptotic values are added in horizontal dashed lines, and maximum damping frequency is added in vertical dashed lines for both RKU model and Guyader's model.

frequency range where the shearing effect of the high-damped constrained core layer is maximum.

For the equivalent rigidity, the three models give very similar results up to the high frequencies (around $10 \mathrm{kHz}$ for SOFT plate in figure 1, and $100 \mathrm{kHz}$ for SPS plate in figure 2). Up to these frequencies, the model of Guyader and the Lamb waves model are perfectly matching whereas the RKU model tends to overestimate slightly the rigidity on the transition zone (governed by shearing of the constrained layer). At higher frequencies, the equivalent rigidity computed with the Lamb waves model falls quickly tending to zero whereas the two other models ignore the through-thickness deformation of the multilayer giving results that tend to the asymptotic value (sum of the flexural rigidities of the structural components as if they were not interconnected). It is worth recalling that the Lamb waves model is exact in that it does not make an assumption with respect to the frequency range in which it is valid. This rapid drop of rigidity occurring at very high frequency is due to the change of wave type in the multilayer, in particular the appearance of breathing modes (out-of-phase flexural motion of the skins of the multilayer). At high frequencies, the wavelength of the bending waves becomes comparable to the thickness of the plate, resulting in physical waves that cannot be represented by the RKU model or Guyader's model (because of the assumptions made for these approaches). The frequency $f_{\mathrm{S} 0}$ of this first breathing resonance (often called symmetric SO-mode resonance in the literature) can be easily estimated considering two equivalent masses $m_{1}$ and $m_{3}$ attached by an equivalent spring of stiffness $k_{2}$, that 
is:

$$
f_{\mathrm{S} 0}=\frac{1}{2 \pi} \sqrt{\frac{k_{2}\left(m_{1}+m_{3}\right)}{m_{1} m_{3}}}=\frac{1}{2 \pi} \sqrt{\frac{E_{2}}{h_{2}} \frac{\rho_{1} h_{1}+\rho_{3} h_{3}}{\rho_{1} h_{1} \rho_{3} h_{3}}}
$$

For the SOFT plate $f_{\mathrm{S} 0} \approx 52.3 \mathrm{kHz}$ which corresponds precisely to the zone where equivalent rigidity of the multilayer begins to vanish (see figure 1). For the SPS plate, the first breathing mode appears at a much higher frequency $(125.3 \mathrm{kHz})$; again, the equivalent rigidity falls when frequency reaches these breathing modes (see the zoomed portion above $100 \mathrm{kHz}$ at the middle of figure 2).

Focusing on the equivalent loss factor, the three models also give similar results for frequencies lower than the first breathing mode frequency. The low- and highfrequency asymptotic values of the equivalent loss factor are well predicted with a very important increase of damping on the optimum-shearing zone. As for the rigidity, the model of Guyader and the Lamb waves model give exactly the same values throughout the whole frequency band (before the change of wave type) whereas the simplified RKU model predictions are not comparable to the two other models in the transition zone: the equivalent loss factor is underestimated. Indeed, as presented in appendix A, the RKU model is a simplified model giving only rough trends as long as it considers only the dissipation through shear motion of the damping layer 2 neglecting for example shear deformation of the skin or membrane effects, on the contrary of more complex models such as the one of Guyader or the exact Lamb waves model). As it could have been predicted, it is worth noticing that these underestimations of loss factor and overestimation of rigidity are less severe when RKU hypotheses are being met (which is the case for the SOFT plate with a much lower Young's modulus of layer 2 ; results shown in figure 1), than for the other multilayer example (SPS plate, figure 2). Finally the increase of equivalent damping computed by the Lamb waves model in the very high frequency domain is due here again to through-thickness deformation when out-of-phase skin motion exists; we will not comment further on the very high frequency behavior as long as it is not the purpose of this article (we will focus in the rest of our study on frequencies lower than the symmetric SO-mode resonance). If needed one can refer for example to Shorter's article [14] for improved study of the different wave types of viscoelastic laminates.

To sum up this theoretical part, the analytical comparisons clearly show the benefits of using the model developed by Guyader for three-layered plates and for frequency where no out-of-phase flexural motion of the skins exists. Furthermore the Guyader's model needs about 0.5 seconds of computation time on a standard computer to compute the solution for the SPS plate for example, whilst the Lamb waves model needs hours due to the inefficient searching for a vanishing determinant as function of both frequency and (complex) wavenumber (thus searching in a 3D space). Guyader's model is much more efficient, whilst its accuracy in the frequency range below the SO-mode resonance is equally good in comparison with the Lamb waves model. The RKU model is also quasi instantaneous such as the 


\begin{tabular}{|c|c|c|}
\hline & SOFT plate & SPS plate \\
\hline Asymptotic low-frequency equivalent Young's modulus [GPa] & 122.7 & 150.4 \\
\hline Asymptotic high-frequency equivalent Young's modulus [GPa] & 49.0 & 2.1 \\
\hline $\begin{array}{l}\text { Maximum loss factor } \eta_{\mathrm{eq}}[-] \\
\text { (Guyader's model and Lamb waves model) }\end{array}$ & 0.015 & 0.041 \\
\hline $\begin{array}{l}\text { Maximum loss factor } \eta_{\text {eq }}[-] \\
\text { (RKU model) }\end{array}$ & 0.014 & 0.025 \\
\hline $\begin{array}{l}\text { Maximum damping frequency }[\mathrm{Hz}] \\
\text { (Guyader's model and Lamb waves model) }\end{array}$ & 503.0 & 8022.0 \\
\hline $\begin{array}{l}\text { Maximum damping frequency }[\mathrm{Hz}] \\
\text { (RKU model) }\end{array}$ & 566.3 & 9002.9 \\
\hline First breathing mode frequency $f_{\mathrm{S} 0}[\mathrm{~Hz}]$ & $52.3 \times 10^{3}$ & $125.3 \times 10^{3}$ \\
\hline
\end{tabular}

Table 2

Relevant analytical results obtained for SOFT and SPS plates.

model of Guyader but overestimates the equivalent rigidity and underestimates the equivalent damping as seen previously. Hence the model of Guyader will be further considered for comparison with experimental measurements performed on the SPS plate over the $[0-20 \mathrm{kHz}]$ frequency domain.

\section{Experimental characterization of material properties}

Four experimental procedures have been used to estimate the material properties of a $300 \times 400 \times 1.05 \mathrm{~mm}^{3}$ SPS multilayer plate sample. The SPS plate is presented in Figure 3. The layer thicknesses reported (and also listed in Table 1) correspond to average values determined using optical microscope images of the plate's cross section. Also, sheets of the polymer layer alone were available (see Figure $3 \mathrm{~b}$ left) so that its density could be determined by measuring and weighting large specimens. Before presenting the results and comparing them to the analytical predictions, we detail the assessment procedures (theories and experimental setups).

\subsection{Wide frequency band behavior}

A typical frequency response (Acceleration/Force) for the SPS plate obtained from impact hammer excitation (see section 3.2.1 for the experimental protocol) is presented in Figure 4 (for the frequency band $[0-3] \mathrm{kHz}]$ ). The low frequency domain is characterized by distinct resonance peaks and the strong modal character of the 
(a)
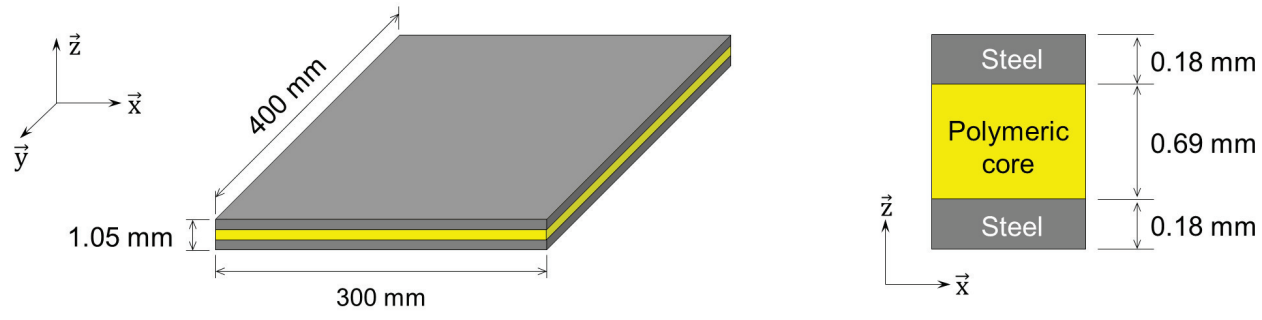

(b)
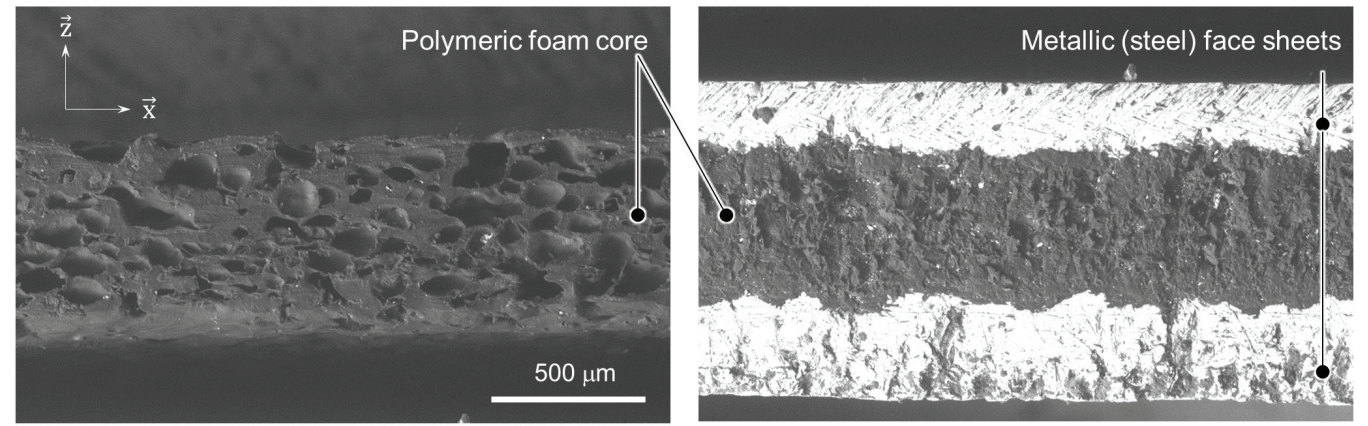

Figure 3. (a) Schematic illustration of the three-layered sandwich plate under study. (b) Optical microscope observation of the through-thickness cross sections of real samples: left - polymer core layer; right - SPS sandwich composite.

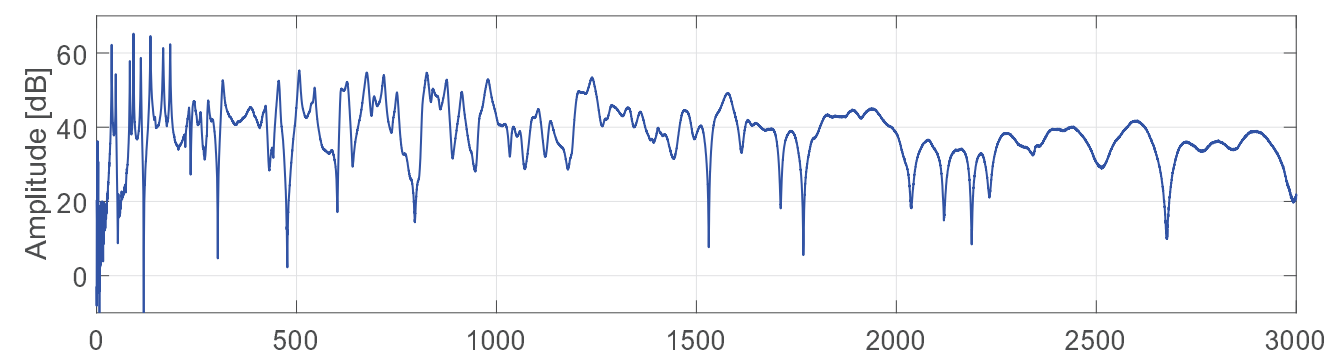

Figure 4. Typical frequency responses "Acceleration/Force" (Fourier spectrum) for the SPS plate, obtained by impact-hammer excitation (see section 3.2.1 for the experimental protocol).

vibratory behavior (here up to around $700-800 \mathrm{~Hz}$ ). The modal analysis and the identification of the Young's modulus in low frequency domain will be presented in section 3.2.1. When the frequency increases, the traditional modal identification methods cannot be used anymore: the damping increases, resonances are thus less pronounced, the modes overlap so that the frequency-response tends to a smooth curve. The ESPRIT [27,10] algorithm will be used to identify modal loss factor for this mid-frequency domain in section 3.2.2. Finally in the high-frequency domain (here above $2.5 \mathrm{kHz}$ ), the vibration can be described as a diffuse wavefield and modal approaches are unsuitable. Thus, the estimation of the complex Young's modulus for wide frequency band will be achieved using two methodologies (up to $20 \mathrm{kHz}$ ): CFAT (section 3.2.3) and Hankel (section 3.2.4). 


\subsection{Assessment procedures (theories and experimental setups)}

\subsubsection{Modal analysis}

To begin, a modal analysis of the plate is performed in order to estimate the first modal frequencies, loss factors and mode shapes. A pseudo-impulse force is applied by means of a small impact hammer (P.C.B. Piezotronics 086E80) on a rectangular mesh of $9 \times 11$ points spanning the whole $300 \times 400 \mathrm{~mm}^{2}$ surface of the plate. The mesh is regularly spaced, resulting in spacings between two consecutive points of $37.5 \mathrm{~mm}$ along the width $x$ and of $40 \mathrm{~mm}$ along the length $y .$. Boundary conditions are kept as close as possible to free-free, by suspending the plate from one of its corner (with rubber bands passing through a tiny hole). The acceleration is measured with a lightweight accelerometer (P.C.B. Piezotronics M353B18) fixed on another corner of the plate. Under the chosen boundary conditions, this location is not on any of the nodal lines for the low frequency band considered here. A multi-degree-of-freedom curve fitting method (Rational Fraction Polynomial-Z) is used to estimate modal frequencies, loss factors and mode shapes. In parallel with the experimental modal analysis, a finite-element model (FEM) calculation of the normal modes of an equivalent single layer plate of same dimensions has been conducted with MSC NASTRAN software (see next paragraph for explanations on the choice of the Young's modulus of the equivalent numerical plate). Four-noded isoparametric flat plate elements of $1 \mathrm{~mm}^{2}$ size have been chosen. The modal frequency convergence criterion has been used to select this element size ensuring far enough points per wavelength (more than 100) up to $800 \mathrm{~Hz}$.

Examples of experimental and numerical mode shapes comparisons are given in Figure 5, where numerical mode shapes are extracted on the 99 experimental mesh points. The Modal Assurance Criterion [9] (called 'MAC') is used here to compare the two sets of results. It calculates to what extent a given mode shape fits another one. More precisely, it consists in calculating the spatial correlation between the two functions, as follow:

$$
\operatorname{MAC}\left(\phi_{(m, n)}^{\exp }, \phi_{(p, q)}^{\text {num }}\right)=\frac{\left|\phi_{(m, n)}^{\exp ^{\mathrm{T}}} \phi_{(p, q)}^{\text {num }}\right|^{2}}{\left(\phi_{(m, n)}^{\exp ^{\mathrm{T}}} \phi_{(m, n)}^{\exp _{(m, q}}\right)\left(\phi_{(p, q)}^{\text {num }} \phi_{(p, q)}^{\text {num }}\right)}
$$

where $\phi_{(m, n)}^{\exp }$ is the experimental mode shape of the $(m, n)$ plate mode and $\phi_{(p, q)}^{\text {num }}$ is the numerical mode shape of the $(p, q)$ plate mode (extracted on the experimental mesh points). If a linear dependence exists between the two functions (identical mode shapes), the MAC value will be near to one. If they are linearly independent (mode shapes differ), the MAC value will be small, close to zero.

As presented in Figure 5, the MAC values are close to $100 \%$ for the first lowfrequency modes and generally decreases with the modal orders. Indeed, values are directly dependent on the experimental mesh size compared to the wavelength. 


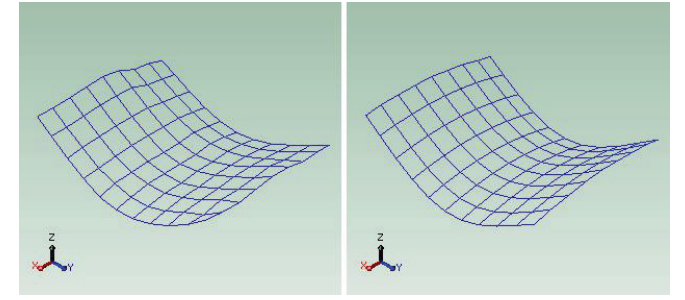

(a) $f_{(0,2)}=47.9 \mathrm{~Hz} ; \mathrm{MAC}=97 \%$; $E_{48 \mathrm{~Hz}}=162 \mathrm{GPa}$

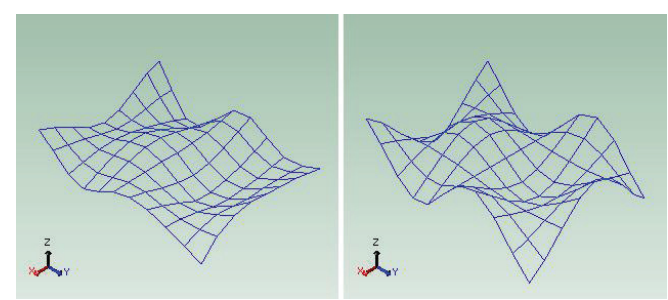

(c) $f_{(2,3)}=276.9 \mathrm{~Hz} ; \mathrm{MAC}=57 \%$; $E_{277 \mathrm{~Hz}}=140 \mathrm{GPa}$

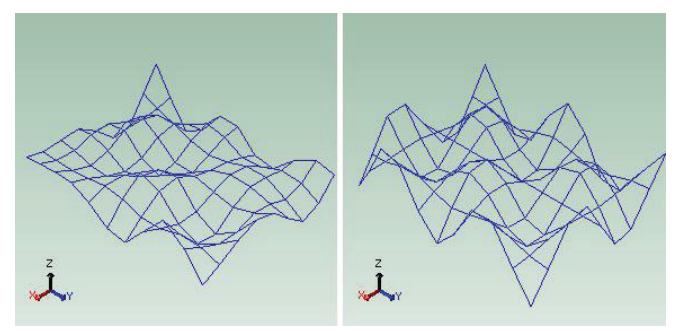

(e) $f_{(3,4)}=535.5 \mathrm{~Hz} ; \mathrm{MAC}=57 \%$; $E_{536 \mathrm{~Hz}}=120 \mathrm{GPa}$

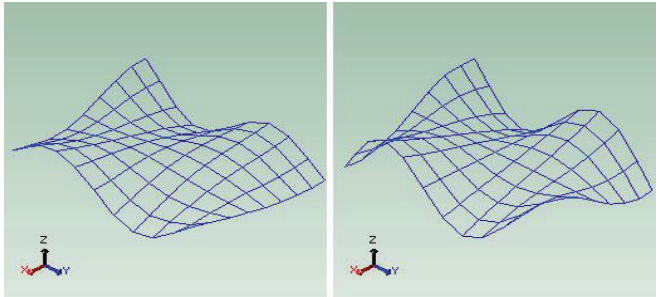

(b) $f_{(1,3)}=165.7 \mathrm{~Hz} ; \mathrm{MAC}=85 \%$; $E_{166 \mathrm{~Hz}}=136 \mathrm{GPa}$

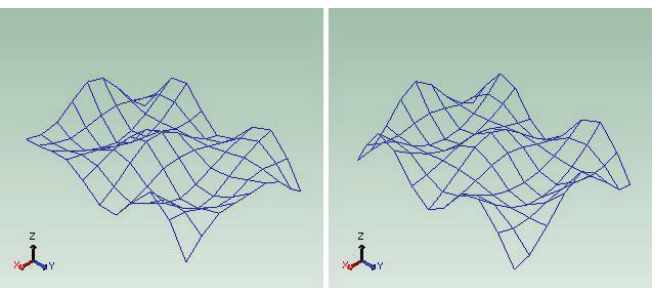

(d) $f_{(2,4)}=385.9 \mathrm{~Hz} ; \mathrm{MAC}=66 \%$; $E_{386 \mathrm{~Hz}}=128 \mathrm{GPa}$

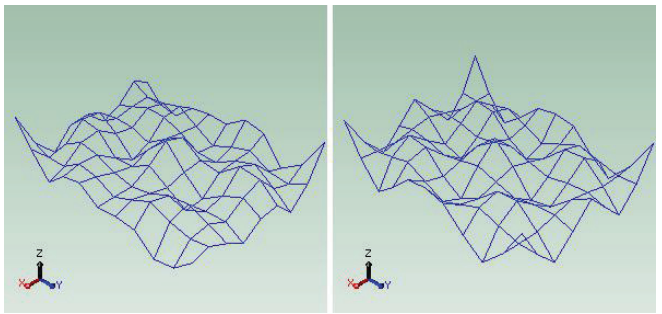

(f) $f_{(4,4)}=718 \mathrm{~Hz} ; \mathrm{MAC}=44 \%$; $E_{718 \mathrm{~Hz}}=110 \mathrm{GPa}$

Figure 5. Comparisons of six experimental (left figure) and numerical (right figure) mode shapes of the SPS plate in the low-frequency domain (numerical mode shapes are extracted on the experimental mesh). For each mode, the MAC value and identified equivalent Young's modulus (by matching FEM estimation to measured modal frequency) are given in caption.

In this study, with a $4 \mathrm{~cm}$ distance between two consecutive excitation points, the modal identification has been successfully obtained up to $800 \mathrm{~Hz}$, with MAC generally higher than $60 \%$. Thanks to this experimental/numerical comparison, a first equivalent Young's modulus estimation of the three-layered SPS plate has been conducted as follow: for each identified mode, the numerical Young's modulus is adjusted so that the numerical modal frequency fits the corresponding experimental one, all other parameters remaining constant (dimensions, thickness and density of the equivalent FEM plate are fixed to the measured values; the Poisson's ratio $\nu$ is arbitrarily set to 0.34). The identified frequency dependent equivalent Young's moduli $E_{\text {eq }}$ are presented in Figure 12 with corresponding MAC values: red points (•) for estimations with highest MAC close to 100\%, green circles (०) for MAC around $70 \%$, and blue asterisks $(*$ ) for MAC lower than $60 \%$. The equivalent loss factors $\eta_{\mathrm{eq}}$ estimated from the modal analysis are presented in Figure 13 in red 
circles (०). Discussion on these results is done in section 4.

\subsubsection{High-resolution modal analysis (ESPRIT algorithm)}

In order to identify the equivalent loss factors of the multilayer plate at higher frequencies, a second approach is used. It consists on a high-resolution modal analysis technique [10] based on ESPRIT algorithm [27] . This high-resolution method assumes that the signal $s(t)$ is a sum of complex exponentials $x(t)$ (the modal signal to be determined) and white noise $\beta(t)$ :

$$
s(t)=x(t)+\beta(t)=\sum_{k=1}^{K} a_{k} \mathrm{e}^{-\alpha_{k} t} \mathrm{e}^{\mathrm{j}\left(2 f_{k} t+\phi_{k}\right)}+\beta(t)=\sum_{k=1}^{K} b_{k} z_{k}^{t}+\beta(t)
$$

where $K$ is the number of complex exponentials, $b_{k}=a_{k} \mathrm{e}^{\mathrm{j} \phi_{k}}$ are the complex amplitudes (with $a_{k}$ and $\phi_{k}$ the modal amplitudes and phases at the point of interest), and $z_{k}=\mathrm{e}^{-\alpha_{k}} \mathrm{e}^{\mathrm{j} 2 \pi f_{k}}$ the complex poles (with $f_{k}$ the modal frequencies in $\mathrm{Hz}$ and $\alpha_{k}$ the modal damping factors in $\mathrm{s}^{-1}$ ). For exponentially damped sinusoids (the signal model considered here), the rotational invariance property of the signal subspace (or modal subspace) is used to estimate the modal parameters (see Roy et al. [27] for mathematical developments). Modal frequencies and modal damping factors are first derived from the complex poles $z_{k}$ (eigenvalues of the spectral matrix [10]) and complex amplitudes $b_{k}$ are then recovered by means of a least square method. The modal damping factor $\alpha_{k}$ (also called modal decay constant in $\mathrm{s}^{-1}$ ), the modal decay time $\tau_{k}$ (in s) and the modal loss factor $\eta_{k}$ (dimensionless) are related as follows:

$$
\alpha_{k}=\frac{1}{\tau_{k}}=\frac{\eta_{k} \omega_{k}}{2} \quad, \quad \eta_{k}=\frac{\Delta f_{k,-3 \mathrm{~dB}}}{f_{k}}=\frac{\alpha_{k}}{\pi f_{k}}
$$

where $\omega_{k}$ is the modal angular frequency (in rad.s ${ }^{-1}$ ) and $\Delta f_{k,-3 \mathrm{~dB}}$ the half-power modal bandwidth. The dimensions of both modal and noise subspaces must be chosen a priori and the quality of the estimation depends significantly on a proper choice for these parameters. The best choice for the dimension of the modal subspace is the number of complex exponentials actually present in the signal. This number $(K)$ is twice the number of real decaying sinusoids (modes). Prior to the modal analysis itself, an estimate of this number is obtained by means of the ESTER (ESTimation ERror) technique [28] which consists in minimizing the error on the rotational invariance property of the signal subspace spanned by the sinusoids. The block diagram in Figure 6 describes the three main steps of the high-resolution method: (a) reconstruction of the acceleration impulse response $\gamma_{\text {imp }}$ (using the measured acceleration $\gamma_{\text {meas }}$ and the measured force $f_{\text {meas }}$ ), (b) signal conditioning (including band-pass filtering and downsampling) as proposed by Laroche [29], (c) order detection, and (d) determination of modal parameters. More details on the different steps of the method are given in Ege et al. [10], where the method is vali- 


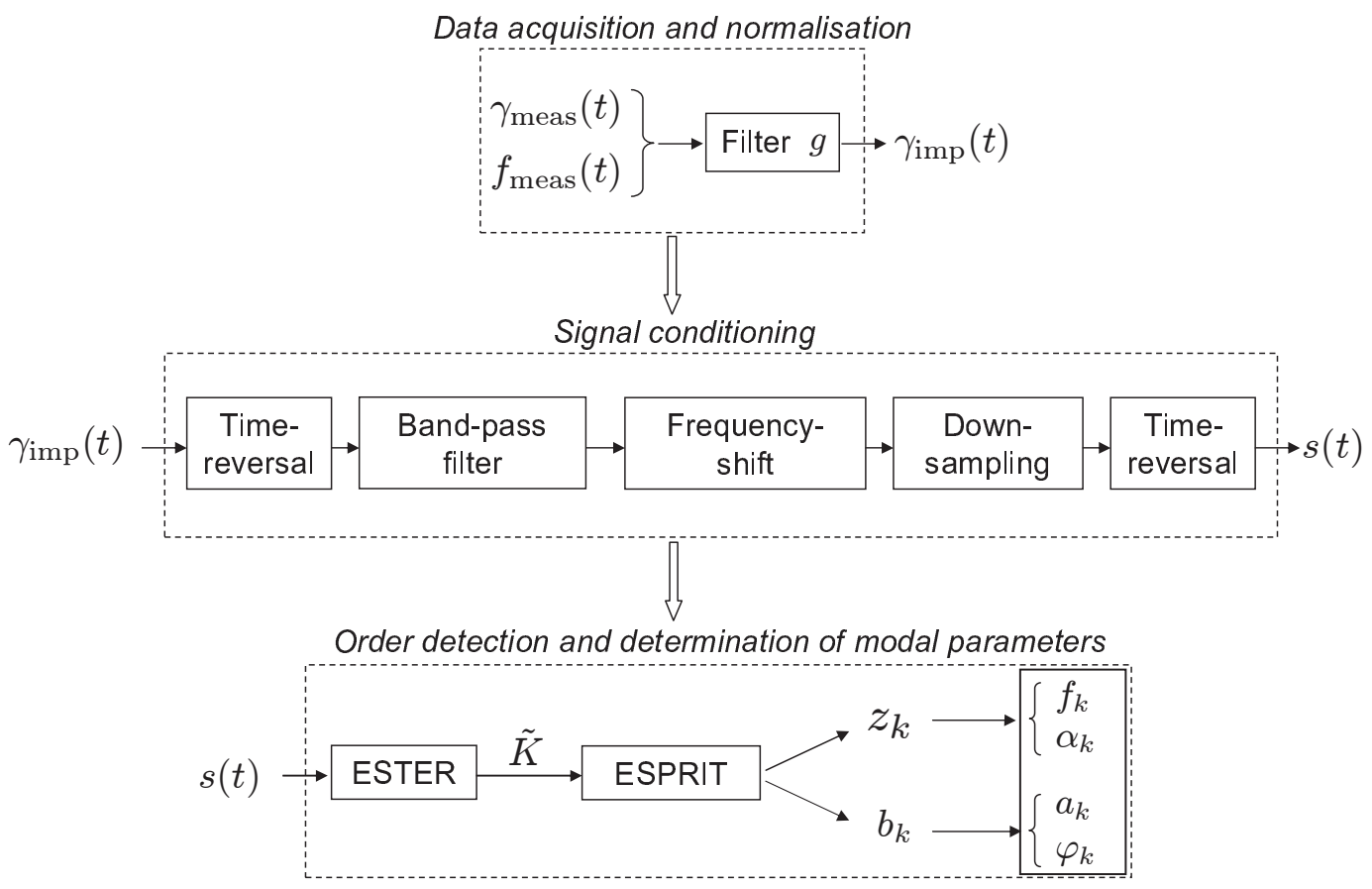

Figure 6. Block diagram of the high-resolution modal analysis method (see Ege et al. [10] for details).

dated on measured and synthesized signals for frequency domain where the Fourier transform meets its limits (due to high modal overlap or poor signal-to-noise ratio). Recently, the method has been also successfully applied on an orthotropic ribbed plate [30], bilayer plates [31], or plate-cavity system [[32],[33]].

The equivalent loss factors of the three-layered SPS plate were accurately estimated using the method described above. The experimental protocol is similar to the previous section. The time signal $s(t)$ analyzed with ESPRIT algorithm corresponds to an excitation made in the vicinity of the accelerometer near one of the corner of the plate. A typical bank-filtering analysis of $s(t)$ is given in Figure 7 where results are plotted for one narrow subband of the mid-frequency domain with four identified modes. Equivalent loss factors have been estimated up to $2.6 \mathrm{kHz}$. Results are plotted in blue points (•) on Figure 13. 

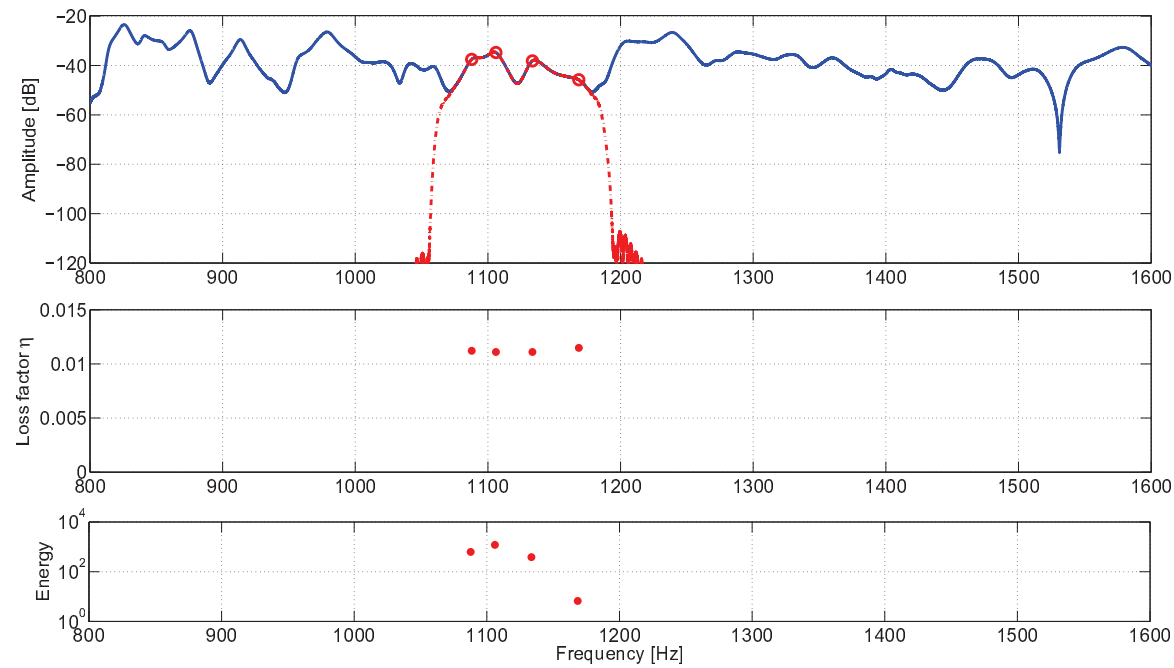

Figure 7. Typical bank-filtering analysis of an impulse response of the SPS plate between 1060 and $1180 \mathrm{~Hz}$. (a) —: Fourier spectrum of the impulse response; - --: amplitude response of a narrow-band filter. $\circ$ marks: estimated modal amplitudes and modal frequencies. (b) $\bullet$ marks: measured loss factors $\eta_{k}$ for the four real modes (the number of complex exponentials $K=8 \quad(=2 \times 4)$ is estimated with ESTER criterion). (c) • marks: Energy of each components. 


\subsubsection{CFAT methodology}

3.2.3.1 Theory The third procedure used to estimate the material properties of the multilayer plate is based upon CFAT methodology [11]. The FAT [34] (Force Analysis Technique) has been published about 20 years ago. The aim of this inverse approach is to recover excitation fields applied on a structure from vibration measurements and from an analytical form of its equation of motion. Typically, for thin homogeneous plates, the following equation is used at the angular frequency $\omega$ :

$$
D \nabla^{4}[w(x, y)]-\rho h \omega^{2} w(x, y)=p(x, y)
$$

where $D=\frac{E(1+\mathrm{j} \eta) h^{3}}{12\left(1-\nu^{2}\right)}$ is the complex rigidity of the plate and $\rho h$ its mass per unit area (that are both known a priori), $w(x, y)$ and $p(x, y)$ are the displacement and load fields, respectively. The displacement $w(x, y)$ is measured on a regular sampling mesh (sampling step $\delta$ ), and its fourth order spatial derivative is estimated using a corrected finite differences scheme CFAT [35], allowing the estimation of the load field from other quantities. The approach has been recently extended to application cases for which the structural parameters $D$ and $\rho h$ are unknown [36,11]. In these cases, the standard method is applied to areas of the structure that are $a$ priori known to be free of excitation. Equation 9 is thus used with $p(x, y)=0$ :

$$
\frac{D}{\rho h}=\frac{\omega^{2} w(x, y)}{\tilde{\nabla}^{4}(x, y)},
$$

where $\tilde{\nabla}^{4}(x, y)$ is an estimation at point $(x, y)$ of the bilaplacian of the displacement field using a corrected finite differences approach [11]. Equation (10) gives an estimation of the parameter at the position $(x, y)$. If it is assumed to be spatially constant (as it is the case here), the parameter can be estimated jointly for all positions of a measurement grid using a Least Squares estimator (see [11] for details). The finite difference scheme used to estimate $\nabla^{4}[w(x, y)]$ is based on 13 measurement points including the central point where the parameter is estimated. The step of the scheme (noted $\Delta$ ) has been shown to give optimal results when lying in the range $\left[\lambda_{\mathrm{N}} / 4, \lambda_{\mathrm{N}} / 2\right]$, with $\lambda_{\mathrm{N}}$ the natural flexural wavelength [37], but for practical reasons has also to match the measurement grid. It is possible, using decimation, to choose a scheme step equal to a multiple of the grid step $(\Delta=n \delta, n \in \mathbb{N}$ ), or even a multiple of the grid step $\times \sqrt{2}(\Delta=n \delta \sqrt{2}, n \in \mathbb{N}$, using a $\pi / 4$ rotation of the scheme) as described in [37]. The scale factor, equal to the ratio between the scheme and grid steps $\Delta / \delta$, is thus chosen between a set of discrete values $\{1 ; 1.4 ; 2 ; 2.8 ; 3 ; 4 ; 4.24 \ldots\}$, and this choice is guided at each frequency by the natural flexural wavelength of the structure. This operation is quite simple for the standard use of CFAT because the flexural wavelength is a priori known. However, when using CFAT to identify the structural parameters, the flexural wavelength is unknown. A possibility is to estimate $D / \rho h$ for various scale factors, and to analyze results with the knowledge that consecutive scale factors should give correct 
results in overlapping frequency ranges. Alternatively, the natural wavelength can be first roughly estimated using 2D FFT for instance, and then used to select the scale factor to be used in CFAT.

Finally, the Young's modulus and loss factor can be obtained as follows:

$$
E=\Re\left(\frac{D}{\rho h}\right) \frac{12 \rho h\left(1-\nu^{2}\right)}{h^{3}} \quad, \quad \eta=\frac{\Im\left(\frac{D}{\rho h}\right)}{\Re\left(\frac{D}{\rho h}\right)}
$$

The mass per unit area $\rho h$ and the thickness $h$ are measured using static metrology systems (weighing machine and caliper) and the Poisson's ratio is arbitrarily taken from standard values.

When applied to homogeneous plates, the resulting Young's modulus is expected to be constant in the frequency range of validity of the CFAT method. When applied to composite panels, Equation (9) can still be used if the vibrational behavior of the plate is assumed to be governed by flexural waves. However, the structure being composed of several layers of materials with different moduli, the relationship between the rigidity $D$ and Young's modulus is not valid anymore. A Young's modulus can still be estimated using Equation (11), but it corresponds to the Young's modulus of an equivalent homogeneous plate of same thickness and mass per unit area, that would have exhibited the same vibrational displacement field at one given frequency. This Young's modulus, called the equivalent Young's modulus, is not expected to be independent of the frequency anymore.

3.2.3.2 Experimental implementation The SPS plate is freely suspended to a frame, and a shaker (driven by a white noise generator) is fixed at an arbitrary position on one edge of the plate (see figure 8). The velocity field of a part of the plate, free of any excitation, is measured using a scanning laser vibrometer. The scanning area is $11 \times 11.6 \mathrm{~cm}$, with a step of about $2.9 \mathrm{~mm}$ (that is to say a total of 1560 measurement points).

The normalized measured velocity field is drawn in figure 9 for few discrete frequencies. It is clear qualitatively that the wavelength decreases when the frequency increases, as expected. This trend is investigated quantitatively in the following using the CFAT procedure.

Measurements are processed as described in section 3.2.3.1. The $D /(\rho h)$ parameters obtained for few scale factors are drawn in Fig. 10. As expected, results using different scale factors are overlapping over the frequency ranges, in which the estimation is thought reliable. 


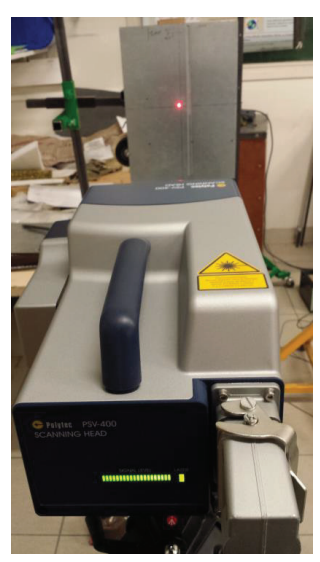

(a)

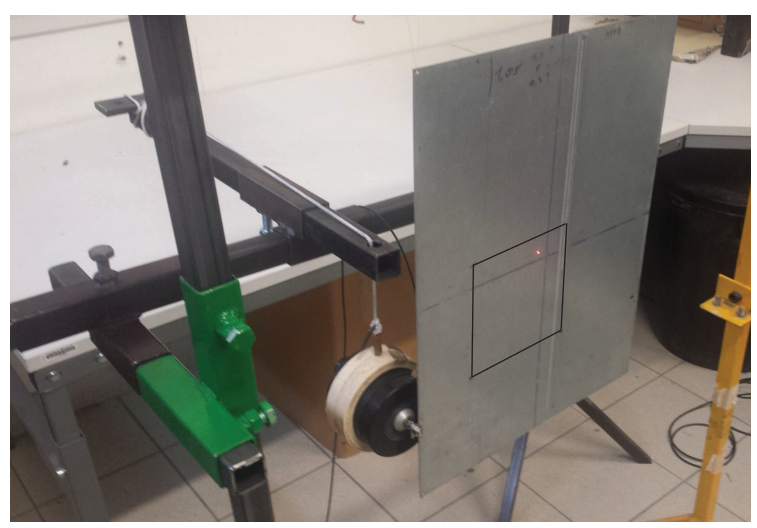

(b)

Figure 8. Measurement set-up for the wave based characterization methodologies (CFAT and Hankel). (a) Laser vibrometer and plate. (b) Plate hanging on thin wires, excited by mini shaker. The black line on the plate indicates the measurement area.
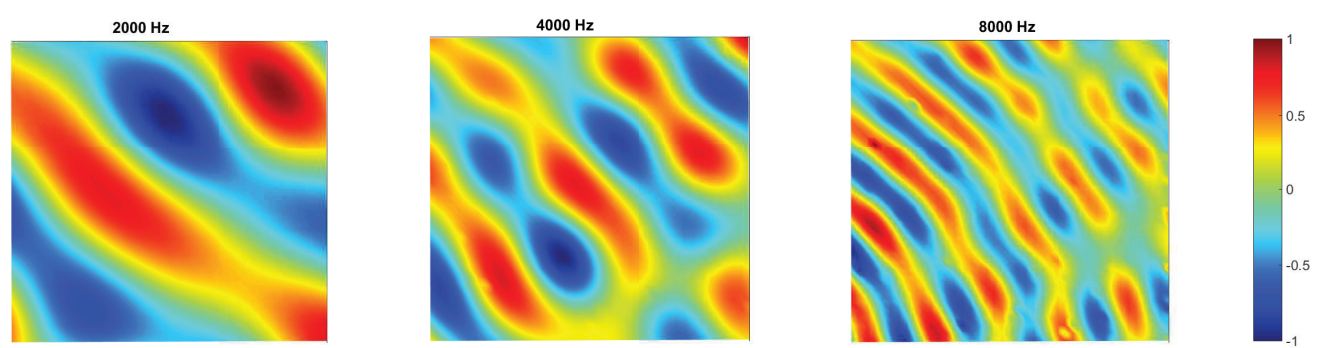

Figure 9. Normalized velocity fields measured at 2(left), 4(center) and 8(right) kHz. The scanning area is $11 \times 11.6 \mathrm{~cm}$.

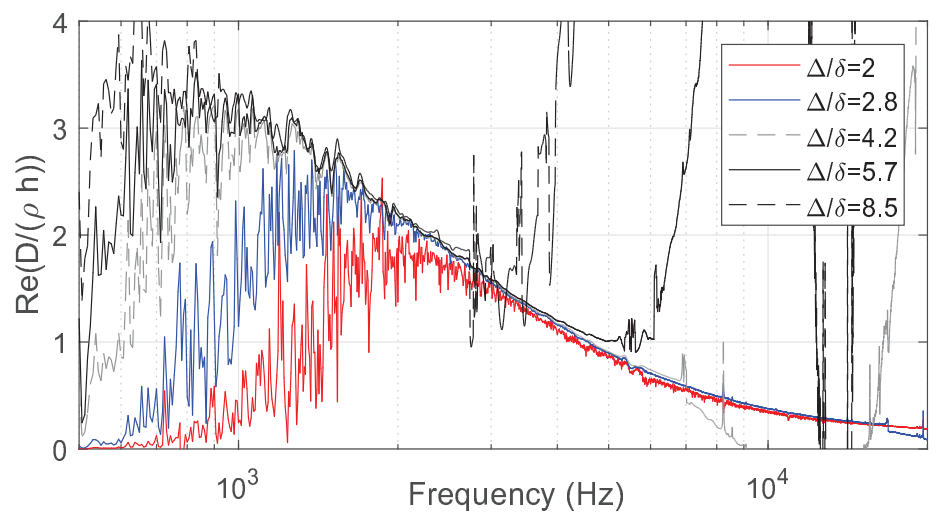

Figure 10. Real part of $D /(\rho h)$ estimated using CFAT with different scale factors $\Delta / \delta$.

A refined approach is implemented in order to obtain a single estimator at each frequency. The natural wavenumber $k_{\mathrm{N}}$ is roughly estimated using a standard maximization of the 2D FFT of the vibration field at one given frequency. The operation is repeated for all frequencies, and a regression is applied in order to obtain a smooth curve of the natural wavenumber as a function of the frequency. The regres- 
sion is obtained by fitting the cloud of points in the frequency-wavenumber plane on a curve with the following shape

$$
k_{\mathrm{N}}(f)=\alpha \sqrt{f}+\beta f
$$

where $\alpha$ and $\beta$ are obtained in the least squares sense. The cloud of points resulting from the 2DFFT maximization at each frequency, as well as the regression curve are drawn in Fig. 11a (left). The regression curve of $k_{\mathrm{N}}$ is then used to determine the

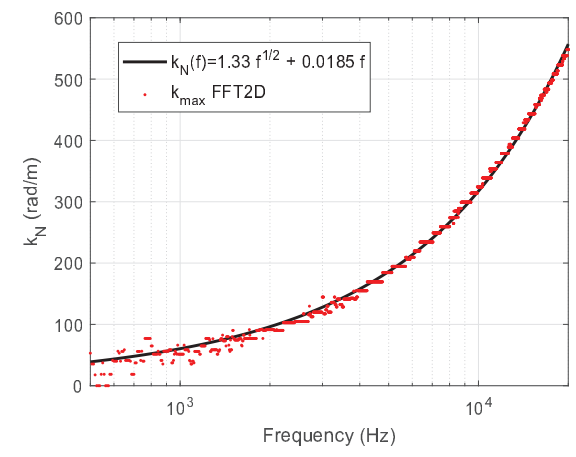

(a)
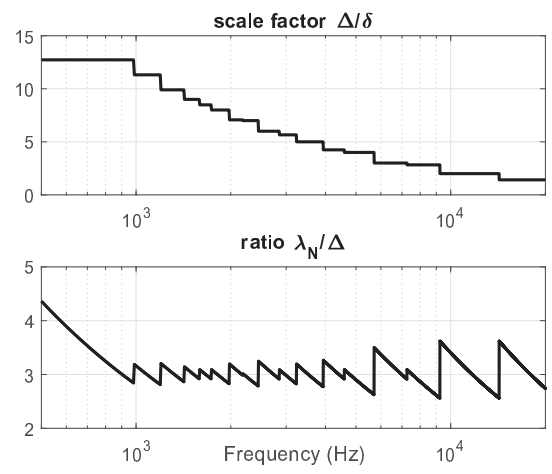

(b)

Figure 11. Rough estimation of $k_{\mathrm{N}}$ and resulting selection of the scale factor used for CFAT. (a) 2DFFT-based estimation of $k_{\mathrm{N}}$ (red dots) and fitted regression (black line). (b) Selected scale factor (top), and ratio between $\lambda_{\mathrm{N}}$ and $\Delta$ (bottom).

scale factor, so as to ensure $\left[\lambda_{\mathrm{N}} / 4<\Delta<\lambda_{\mathrm{N}} / 2\right]$. Practically speaking, the choosen scale factor is the closest one to $\lambda_{\mathrm{N}} / 3$ (see Fig. $11 \mathrm{~b}$, right).

Finally, considering for the SPS plate $\rho h=3.21 \mathrm{~kg} \cdot \mathrm{m}^{-2}$ and $h=1.05 \mathrm{~mm}$, the Young's modulus and loss factor estimated using Eq. (11) are added on figure 12 and 13.

\subsubsection{Material parameter fit by means of a Hankel's functions image source model}

The fourth procedure to estimate the equivalent material properties of the multilayer plate is a wave correlation technique comprising an image source model that uses Hankel's functions, as described in [12]. The model describes the dynamics of an equivalent plate of finite dimensions with homogeneous material properties. Searching for an optimum fit of this image source model on to the measurement data, the equivalent material properties of the multilayer plate are obtained.

In more details, for a homogeneous plate with infinite lateral dimensions, the Green's function $G_{\infty}$ is given by:

$$
G_{\infty}\left(x-x_{0}, y-y_{0}\right)=\frac{1}{8 k_{\mathrm{f}}^{2} D}\left(H_{0}^{(1)}\left(k_{\mathrm{f}} r\right)-H_{0}^{(1)}\left(\mathrm{j} k_{\mathrm{f}} r\right)\right)
$$


where $H_{0}^{(1)}$ is the cylindrical Hankel's function of the first kind of order $0, x$ and $y$ are the coordinates on the plate, $x_{0}$ and $y_{0}$ are the coordinates of the point of excitation, $r=\left\|\left(x-x_{0}, y-y_{0}\right)\right\|$ is the source-to-receiver distance, $k_{\mathrm{f}}$ is the flexural wave number defined by $k_{\mathrm{f}}=\left(\omega^{2} \rho h / D\right)^{1 / 4}, D$ is the dynamic flexural rigidity defined by $D=E h^{3} /\left(12\left(1-\nu^{2}\right)\right), E$ is Young's modulus, $\rho$ the density, $h$ the thickness of the plate, $\nu$ Poisson's ratio and $\omega$ denotes the exciting angular frequency.

The Green's function of a finite plate can be approximated by means of the image source method, summing up the responses of the individual sources. Provided that frequency and/or damping of the plate are high enough, a limited number of image sources suffices to describe the dynamic response of the finite plate, including the reflected wave fields from its edges. This makes this technique especially suited for frequencies with a high modal density and a reasonable amount of damping.

The (complex valued) image source strengths are estimated from a fit on the experimental data, using informations regarding the positions of the image sources (which follow from the geometry of the plate and the point of excitation). Assuming that $N$ image sources are sufficient the response $\tilde{w}$ of the finite plate can then be written as:

$$
\tilde{w}(x, y, \omega)=\sum_{n=1}^{N} \alpha_{n}(\omega) G_{\infty n}(x, y)
$$

where $\alpha_{n}$ is the strength of each image source and $G_{\infty n}$ the Green's function of the $n$ 'th image source. This equation can be written in matrix notation as:

$$
\tilde{\mathbf{w}}=\Phi \alpha
$$

where $\tilde{\mathbf{w}}$ is a vector containing the projected displacements at each measurement point, $\Phi$ is a matrix containing the vectors of the image source Green's functions, $G_{\infty n}$, and $\boldsymbol{\alpha}$ is a vector containing the contribution strengths $\alpha_{n}(\omega)$. The optimum values for vector $\alpha$ are found solving the following minimization problem:

$$
\boldsymbol{\alpha}=\operatorname{argmin}\left(\|\mathbf{w}-\Phi \boldsymbol{\alpha}\|^{2}+\lambda^{2}\|\boldsymbol{\alpha}\|^{2}\right),
$$

where $\mathbf{w}$ are the measured displacements and $\lambda$ is a regularization parameter, giving the following solution for $\alpha$ :

$$
\boldsymbol{\alpha}=\boldsymbol{\Phi}^{+\lambda} \mathbf{w}=\left(\boldsymbol{\Phi}^{*} \boldsymbol{\Phi}+\lambda^{2} \mathbf{I}\right)^{-1} \boldsymbol{\Phi}^{*} \mathbf{w} .
$$

In the present paper, a Bayesian regularization is used (see [12] for details). The source strengths (in amplitude and phase) of the image sources are thus estimated by projecting the measured complex response of the plate onto the image source model. The method is applicable to an arbitrary boundary condition of the plate.

Varying both the real and the imaginary parts of the complex flexural wave number $k_{\mathrm{f}}$ in the image source model (cfr. Eq. 12), an optimum value of $k_{\mathrm{f}}$ is sought for which the error between the predicted response and the measured response is mini- 
mal. From the optimum complex flexural wave number $k_{\mathrm{f}}$ as function of frequency, the (complex) dynamic flexural rigidity $D$ can be estimated from $D=\omega^{2} \rho h / k_{\mathrm{f}}^{4}$. The loss factor can be estimated from $\eta=\Im\left(k_{\mathrm{f}}^{4}\right) / \Re\left(k_{\mathrm{f}}^{4}\right)$. The results obtained using this wave correlation technique comprising an image source model that uses 8 Hankel's functions are shown in figure 12 for the equivalent Young's modulus, and in figure 13 for the equivalent loss factor. Note that the experimental protocol and the measurement dataset are the same as for CFAT (see beginning of section 3.2.3.2).

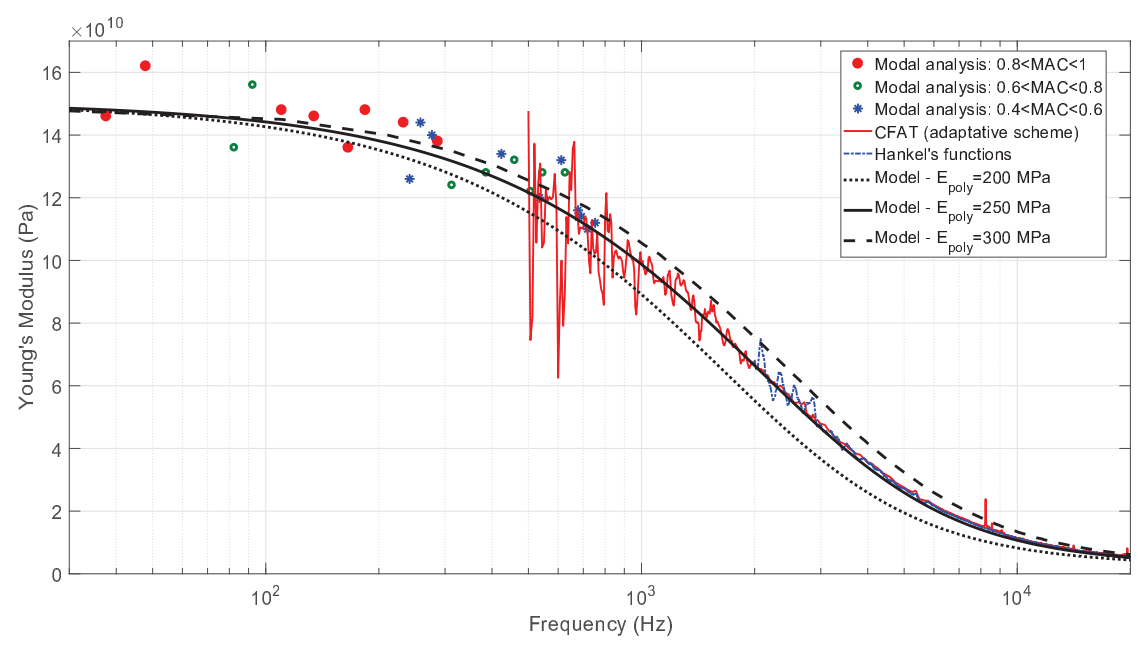

Figure 12. Equivalent Young's modulus $E_{\text {eq }}$ function of the frequency for the steel-polymer-steel (SPS) plate.

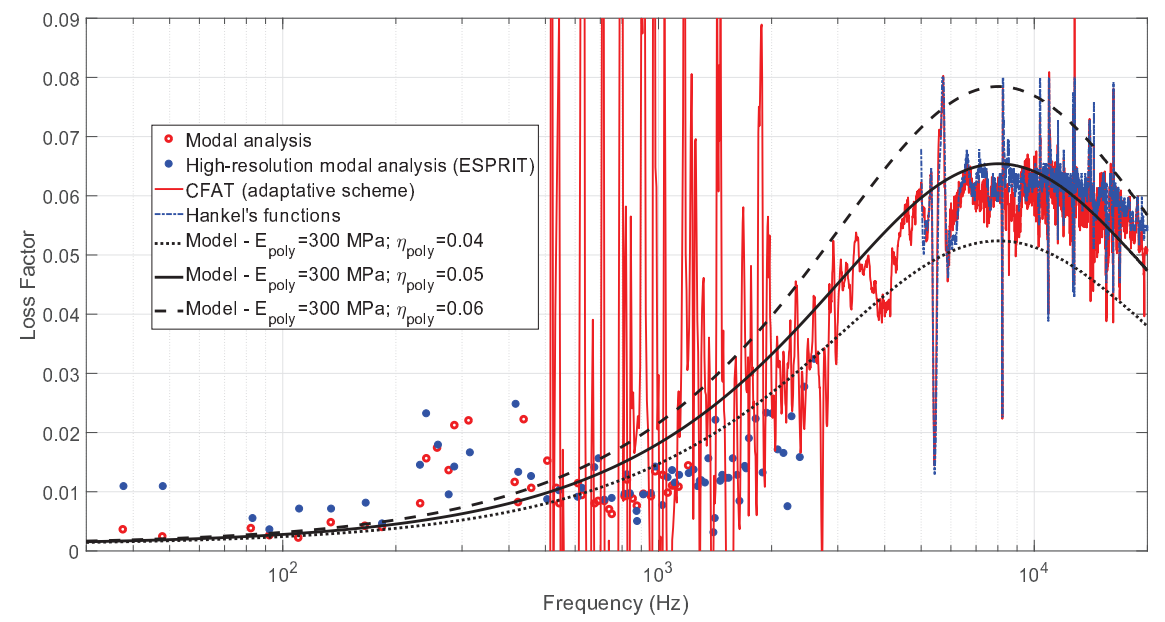

Figure 13. Equivalent loss factor $\eta_{\text {eq }}$ function of the frequency for the steel-polymer-steel (SPS) plate. 


\section{Comparing experimental and analytical results}

In this last section, we compare the experimental results to the analytical predictions allowing the identification of the polymer core complex modulus. Finally the article ends with the comparison of these identified polymer characteristics with DMA measurements/extrapolations based on the time-temperature superposition principle.

\subsection{Equivalent complex modulus of the three-layer and polymer core character- istics identification}

The frequency dependent equivalent Young's modulus and equivalent loss factor have been analytically predicted for the SPS plate using the aforementioned single layer plate model of Guyader. In our study, the major unknowns are the polymer core layer Young's modulus $E_{\text {poly }}$ and loss factor $\eta_{\text {poly }}$; indeed the thicknesses of the layers have been measured, the density has been determined from weight and dimensions measurements, and the Poisson's ratio $\nu$ is arbitrarily set to 0.34 . For the steel skin layers, standard values for the material properties have been selected with a constant value of $10^{-3}$ for the skins loss factors $\eta_{1}$ and $\eta_{3}$ in agreement with measurements done on a homogeneous steel plate from the same manufacturer. Thus, a fitting process has been conducted to identify the polymer core mechanical properties needed to converge to the sandwich experimental estimations. Figures 12 and 13 display the equivalent Young's modulus and the equivalent loss factor respectively as a function of the frequency. Each figures gather the results from the different experimental estimations - modal analysis, ESPRIT algorithm (loss factor estimations), CFAT and Hankel's functions - and the analytical predictions with identified sets of parameters for the polymer core Young's modulus $E_{\text {poly }}$ and loss factor $\eta_{\text {poly }}$.

The identification of these two quantities $E_{\text {poly }}$ and $\eta_{\text {poly }}$ in model of Guyader requires two steps. The fitting process consists in identifying first $E_{\text {poly }}$ by converging to measured equivalent Young's modulus, using the two velocity field measurements in particular (CFAT estimations and Hankel's functions) that cover the frequency range from approximately $1 \mathrm{kHz}$ up to $20 \mathrm{kHz}$. Figure 12 evidences that this value is about $E_{\text {poly }}=250 \mathrm{MPa}$ for frequencies between $[100 \mathrm{~Hz}-$ $1 \mathrm{kHz}$ ] and tends to $E_{\text {poly }}=300 \mathrm{MPa}$ for higher frequencies(see the solid and dashed black curves). With $E_{\text {poly }}$ estimated, $\eta_{\text {poly }}$ is then fitted by comparing the predicted/measured equivalent loss factors. This value has been identified around $\eta_{\text {poly }}=5 \%$ (see the solid black curve on Figure 13). With these identified polymer core mechanical properties, the predicted and experimental estimations of the multilayer equivalent parameters are in very good agreement, with overlapping estimations, both for Young's modulus than loss factor up to very high frequencies. 
It is worth noticing that such experimental results especially for loss factor estimations done from low frequency up to $20 \mathrm{kHz}$ are rare in the literature.

For the equivalent Young's modulus $E_{\mathrm{eq}}$ the tendency is particularly well predicted by the model (see discussion on section 2.4). The value is close to $150 \mathrm{GPa}$ in the low-frequency domain and decreases to around $6 \mathrm{GPa}$ at $20 \mathrm{kHz}$ tending to an asymptotic value (see Figure 12). For the equivalent loss factor $\eta_{\mathrm{eq}}$ (Figure 13), results are also very satisfying, even if the discrepancies in the low frequency domain are higher than for the real part estimation. These discrepancies for the first modes could have for origin the hanging system, or the accelerometer cable, through which a part of the vibratory energy can escape, leading to an overestimation of the damping. Nevertheless, taking apart these fluctuations for the first modes, the experimental loss factor frequency evolution on the whole frequency band matches well the equivalent single layer modelling predictions. In the low frequency part, the damping is small and close to the metal losses (around $0.2 \%$ ), then regularly increases with frequency, reaching high values $\left(\eta_{\mathrm{eq}}=6 \%\right)$ for the frequency band 6-10 kHz, before decreasing to around $5 \%$ at $20 \mathrm{kHz}$. Here again, the increasing of shearing of the dissipative polymer core layer is responsible for this large increase of damping. Such results show the benefits of using sandwich three-layer for vibroacoustics applications in terms of high-damping properties for given frequency domains.

It is worth precising here that for the whole frequency domain of interest, the thin plate equivalent to the multilayer under study vibrates in the subsonic regime. In other terms, in the frequency range $[40 \mathrm{~Hz}-20 \mathrm{kHz}]$, the equivalent flexural wavelengths are always much shorter than the acoustic wavelengths: the coincidence frequency of the equivalent (homogeneous) plate $f_{\mathrm{c}, \mathrm{eq}}=\frac{c_{\mathrm{air}}^{2}}{2 \pi} \sqrt{\frac{\rho_{\mathrm{eq}} h}{D_{\mathrm{eq}}}}$ (with $c_{\mathrm{air}}$ the speed of sound in air) is systematically more than $10 \mathrm{kHz}$ higher than the frequency of interest. Hence there cannot be efficient coupling between flexural waves in the multilayer and waves in air in this frequency range: the added damping due to radiation is neglected.

\subsection{Comparison of the estimated complex Young's modulus of the polymer with DMA measurements}

With the aim of evaluating the validity of our inverse method to identify the polymer core properties, the low frequency viscoelastic properties of the polymer layer was directly measured using Dynamic Mechanical Analysis (DMA). In details, polymer rectangular stripes (10 $\mathrm{mm}$ wide and $60 \mathrm{~mm}$ long) were prepared and loaded in tension under forced periodic strain with a GABO Eplexor DMA apparatus (see Figure 14). Three frequencies $(0.2,1$ and $5 \mathrm{~Hz})$ and temperatures encompassing room temperature $(240 \mathrm{~K}-380 \mathrm{~K})$ were tested. The imposed displacement and measured force signals give access to the complex modulus $E^{*}$ : 

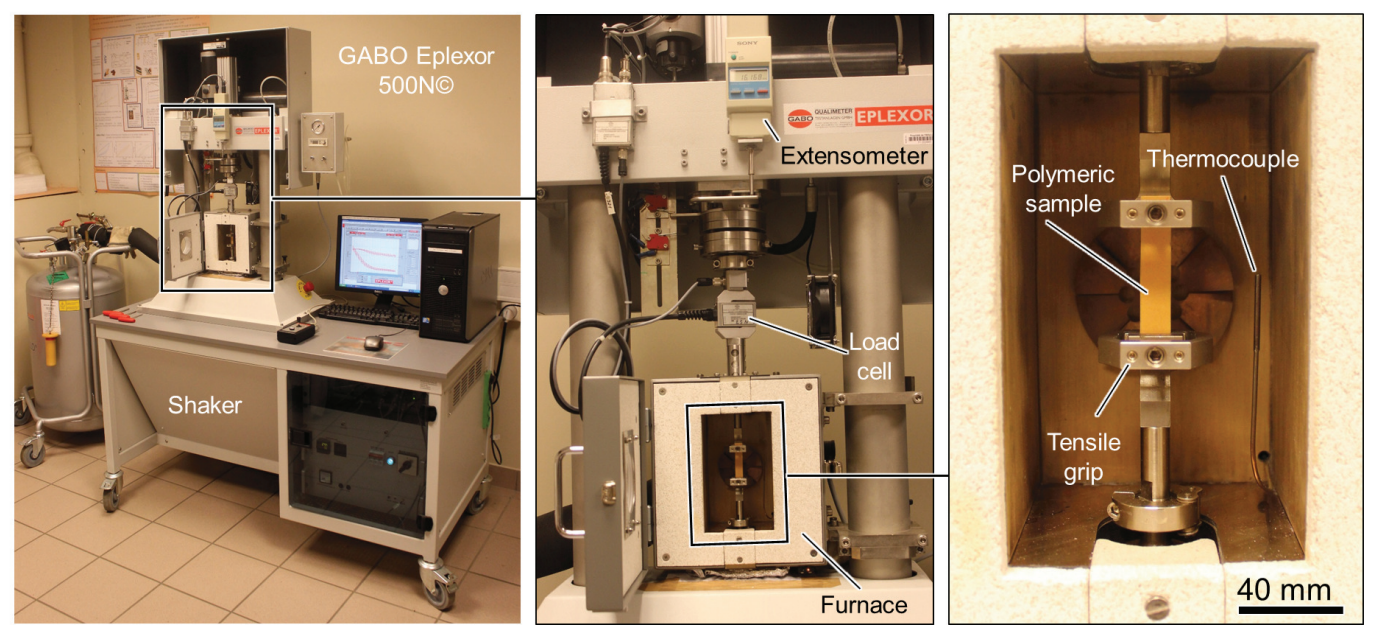

Figure 14. GABO Explexor DMA apparatus with tensile grips (with zoom-in from left to right).

$$
E^{*}=E^{\prime}+\mathrm{j} E^{\prime \prime}=E^{\prime}(1+\mathrm{j} \eta)
$$

with $E^{\prime}$ and $E$ " the storage and dissipative moduli respectively. It is worth noting that $E^{\prime}$ corresponds to the Young's modulus and is also denoted $E$ in this paper. Also, $\eta$ will be further referred as $\tan \delta$ and corresponds to the loss factor.

Both $E^{\prime}$ and $\tan \delta$ are displayed in Figure 15 and a strong temperature and frequency dependencies can be observed: the modulus is seen to decrease as the temperature increases. Conversely, for a given temperature, the modulus increases as the testing frequency increases. More specifically, the isochronal curves seem to simply shift towards higher temperature as the frequency increases, suggesting that the polymeric material can be considered as thermosimple so that time-temperature equivalence principle does apply. Thus, the mastercurve was built following the method proposed by Blachot et al. [38]. The method was implemented in a Matlab routine and the prediction of the polymer complex modulus can be obtained for a wide range of frequencies that cannot be tested experimentally. This is evidenced in Figure 16, where the predicted Young's modulus for frequencies ranging from $10^{-5} \mathrm{~Hz}$ to $10^{10} \mathrm{~Hz}$ are displayed.

The DMA extrapolations can then be used to read the frequency dependence of the Young's modulus at room temperature (296 K). Focusing on the high frequencies values, the polymer modulus $E_{\text {poly }}$ is found to be about $250 \mathrm{MPa}$ at $100 \mathrm{~Hz}$, $273 \mathrm{MPa}$ at $1 \mathrm{kHz}$, and $294 \mathrm{MPa}$ at $10 \mathrm{kHz}$ (see red dashed curve on Figure 17). It is worth noticing that these predictions accurately match the values identified based on the vibratory approach depicted in the previous section.

Similar conclusions can be drawn on the loss factor (see blue dashed curve on figure 17). The high frequencies values gives a polymer loss factor of about $6.5 \%$ 


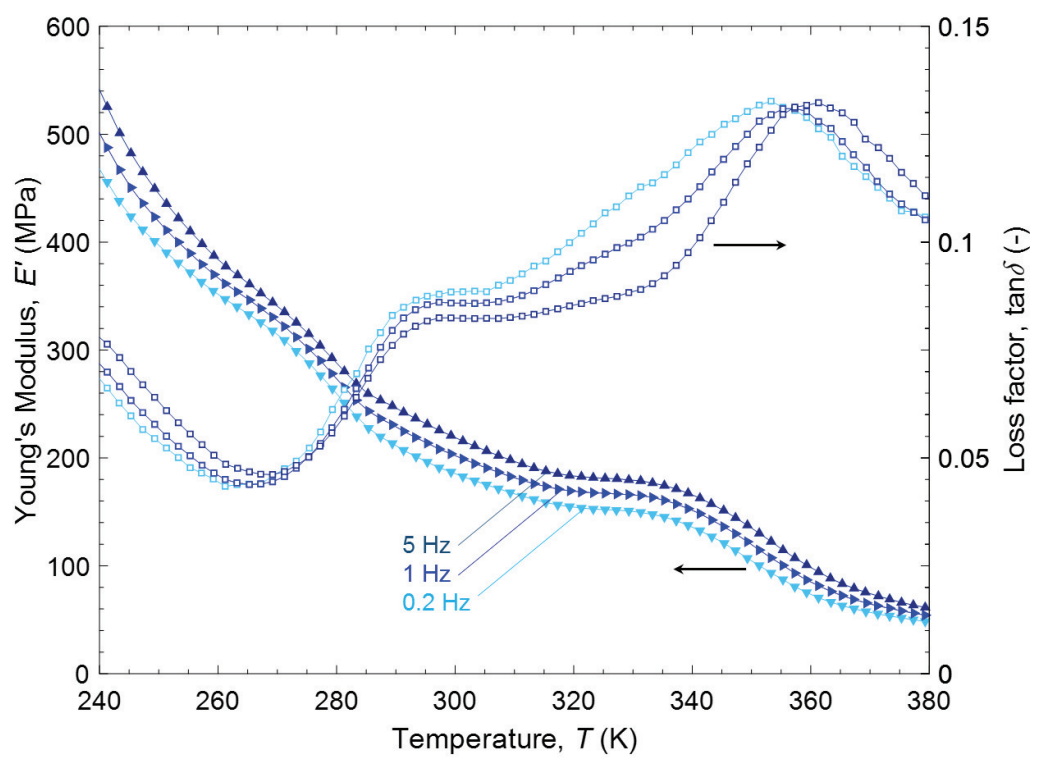

Figure 15. Experimental Young's modulus and loss factor of the polymer core layer as a function of temperature.

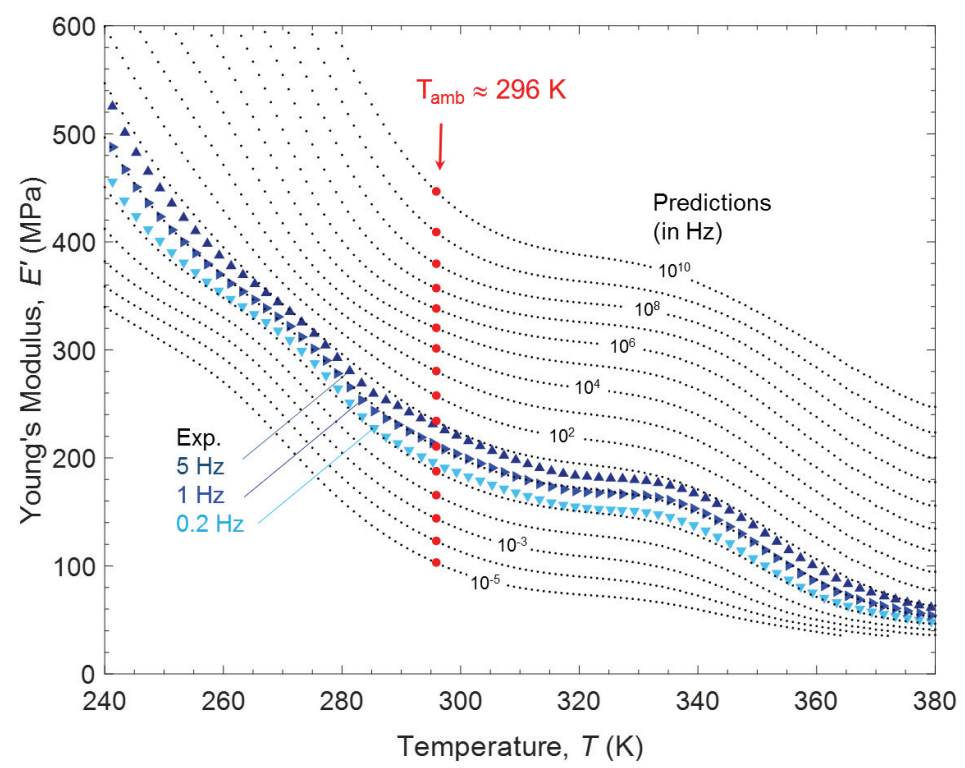

Figure 16. Measured and predicted Young's modulus of the polymer core versus temperature for varying frequencies. The predictions are obtained based on the time-temperature superposition principle.

at $1 \mathrm{kHz}$ and about $5 \%$ at $10 \mathrm{kHz}$. These values match here again the loss factor estimated through vibratory approached for SPS case (5\%, on figure 13). Hence, the accuracy of both estimations is very satisfying for this very high frequency domain, 


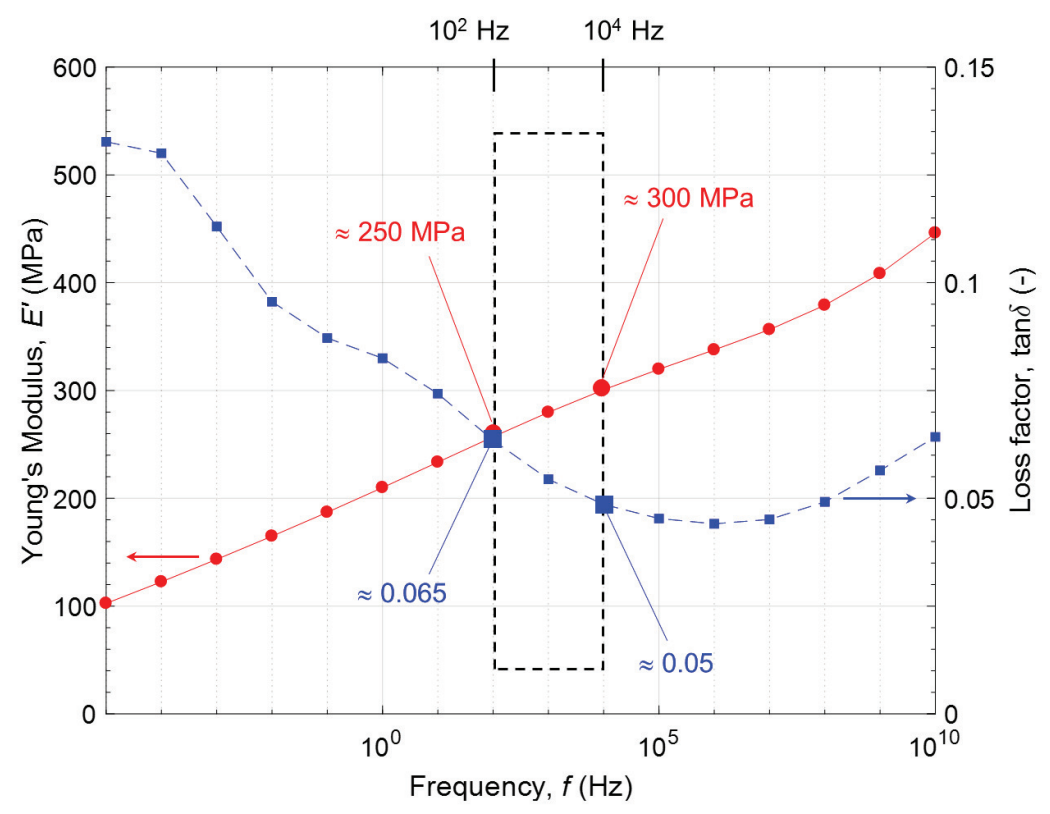

Figure 17. Predicted Young's modulus and loss factor of the polymer core versus frequency at ambient temperature $(296 \mathrm{~K})$.

and demonstrates the validity of the wide-band inverse methodology developed. 


\section{Conclusions}

This paper is focused on the vibrational behavior of sandwich composite plates. Frequency dependent equivalent Young's modulus and equivalent loss factor of a steel-polymer-steel sandwich system are estimated in a broad-band frequency range using different experimental methods. The results are in very good agreement with an equivalent single layer analytical modelling (model of Guyader) based on wave propagation analysis. The comparison with this theoretical approach allows the identification of the frequency dependent complex modulus of the polymer core layer through inverse resolution that matches DMA measurements/predictions performed on the polymer material alone.

There are several perspectives and applications to this work. With the approach presented, optimization of multilayer plates for vibroacoustics applications, with optimized damping properties for given frequency domains, may be easily performed if the precise knowledge of the frequency evolution of the complex modulus of each layer is known. In this respect, an interesting perspective could be to use a recently developed UV irradiation selective technique of Room Temperature Vulcanization silicone elastomeric membrane to achieve spatial patterning of the in-plane viscoelastic properties of the polymeric core of sandwich structure [39] in order to design optimized structured plate.

It could be also challenging for such multilayer systems to experimentally explore frequencies higher than $20 \mathrm{kHz}$, using for example a Nd:YAG pump laser (see a recent work done on that subject up to $50 \mathrm{kHz}$ [40]) or ultrasound exciters; other types of waves would be excited and it still remains very difficult to identify them precisely (in particular the dissipation associated to them) and compare the measurements with predictions (such as the Lamb waves model presented in this work).

Next to that, using the concept of an equivalent plate with frequency dependent material properties representing the dynamics of a sandwich plate system allows a computationally more efficient way to model numerically (for instance using finite element modelling) the structural response of complex structures made from these sandwich plate systems. Indeed, this study shows that without complex composites theories or 3D elements, a relatively simple (2D) Love-Kirchhoff thin plate theory with a frequency dependent equivalent (or apparent) complex modulus may be sufficient for frequency bands where no through-thickness deformation of the multilayer exists. 


\section{A The model of Ross, Kerwin and Ungar}

In what follows below is a brief summary of the simplified model of the dynamics of a constrained layer system being composed of three layers (initially written for beams) developed by Ross, Kerwin and Ungar [19], [20], [21], [24]. Only final expressions are given here (for the plate case); the whole derivations are exposed in the cited articles. The notations used here below are the one introduced by Ungar [21], also reported in Beranek's book [41].

The thicknesses $h_{1}, h_{2}, h_{3}$ and the dimension $H_{31}$ of the three-layered plate are indicated in Figure A.1. The complex Young's modulus of layer $i$ is denoted by $E_{i}^{*}=E_{i}\left(1+\mathrm{j} \eta_{i}\right)$, where $\eta_{i}$ is the loss factor of layer $i(i=1,2,3)$. The complex shear modulus of the second (visco-elastic) layer is denoted by $G_{2}^{*}=\frac{E_{2}^{*}\left(1+\mathrm{j} \eta_{2}\right)}{2\left(1+\nu_{2}\right)}$.

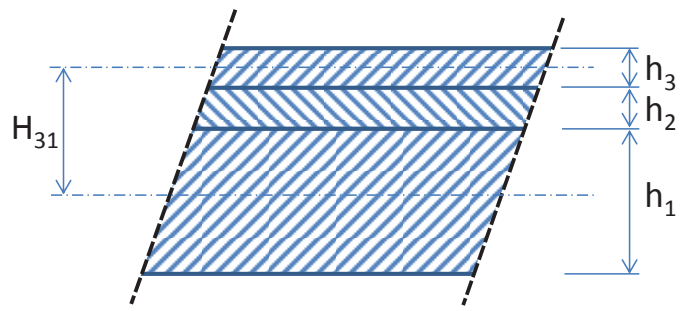

Figure A.1. Three-layered constrained layer plate.

\section{A.1 Equivalent complex flexural rigidity}

The RKU model gives an expression for the equivalent complex flexural rigidity $D^{*}$ of the three-layer as:

$$
D_{\mathrm{eq}}^{*}=\left(D_{1}^{*}+D_{3}^{*}\right)\left(1+\frac{X^{*} Y^{*}}{1+X^{*}}\right)
$$


where

$$
\begin{aligned}
D_{1}^{*} & =\frac{E_{1}^{*} h_{1}^{3}}{12\left(1-\nu_{1}^{2}\right)} \\
D_{3}^{*} & =\frac{E_{3}^{*} h_{3}^{3}}{12\left(1-\nu_{3}^{2}\right)} \\
X^{*} & =\frac{G_{2}^{*} S^{*}}{k^{2} H_{2}} \\
Y^{*} & =\frac{12 H_{31}^{2}}{S^{*}\left(E_{1}^{*} h_{1}^{3}+E_{3}^{*} h_{3}^{3}\right)} \\
S^{*} & =\frac{1}{E_{1}^{*} h_{1}}+\frac{1}{E_{3}^{*} h_{3}}
\end{aligned}
$$

Hence $D_{\text {eq }}^{*}$ depends only on the complex flexural rigidities $D_{1}^{*}$ and $D_{3}^{*}$ of layer 1 and 3, and on two dimensionless parameters introduced by Ungar [21]: $X^{*}$ called shear parameter (function of the wavenumber $k$ ) and $Y^{*}$ called structural geometric parameter. To use the terms of Ungar [41], this shear parameter $X$ is a measure of how well the viscoelastic layer (layer 2) couples the flexural motions of the two structural components (layer 1 and 3).

The real part of $D_{\mathrm{eq}}^{*}$ is the equivalent flexural rigidity of the 3-layered system (from which the equivalent Young's modulus $E=\Re\left(D_{\mathrm{eq}}^{*}\right) \frac{12\left(1-\nu_{\mathrm{eq}}^{2}\right)}{h^{3}}$ can be derived knowing the total thickness $h=h_{1}+h_{2}+h_{3}$ and equivalent Poisson's ratio $\nu_{\mathrm{eq}}=$ $\frac{\sum_{i} h_{i} \nu_{i}}{\sum_{i} h_{i}}$ of the multilayer), whilst the effective loss factor can be calculated by

$$
\eta_{\mathrm{eq}}=\frac{\Im\left(D_{\mathrm{eq}}^{*}\right)}{\Re\left(D_{\mathrm{eq}}^{*}\right)}
$$

Note finally that $D_{\text {eq }}$ is of course function of the flexural wavenumber $k$ through the shear parameter $X$. Hence the correspondent frequency $f$ (for plotting $D_{\text {eq }}(f)$ figure 12 and $\eta_{\text {eq }}(f)$ figure 13 in particular) is given using the dispersion relation of the Love-Kirchhoff theory for the equivalent plate of total thickness $h$ and equivalent density $\rho_{\mathrm{eq}}=\frac{\sum_{i} h_{i} \rho_{i}}{\sum_{i} h_{i}}$ by

$$
f=\frac{1}{2 \pi} \sqrt{\frac{D_{\mathrm{eq}} k^{4}}{\rho_{\mathrm{eq}} h}}
$$


Asymptotic values of the equivalent flexural rigidity can be derived from equation A.1 for low and high frequencies. For small $f$, the wavenumber tends to zero, and $X=\Re\left(X^{*}\right)$ tends to infinity: the three-layered panel exhibits a rigidity $D_{\mathrm{LF}}=\Re\left(D_{\mathrm{LF}}^{*}\right)$ equal to the flexural rigidity of a panel with rigidly interconnected structural components 1 and 3. For high frequencies instead, the wavenumber tends to infinity, and $X$ tends to zero: the equivalent rigidity $D_{\mathrm{HF}}=\Re\left(D_{\mathrm{HF}}^{*}\right)$ is simply the sum of the flexural rigidities of the structural components (as if they were not interconnected). These expressions are given here:

$$
\begin{aligned}
& D_{\mathrm{eq}, \mathrm{HF}}=\frac{E_{1} h_{1}^{3}}{12\left(1-\nu_{1}^{2}\right)}+\frac{E_{3} h_{3}^{3}}{12\left(1-\nu_{3}^{2}\right)} \\
& D_{\mathrm{eq}, \mathrm{LF}}=D_{\mathrm{eq}, \mathrm{HF}}(1+Y)=\left(\frac{E_{1} h_{1}^{3}}{12\left(1-\nu_{1}^{2}\right)}+\frac{E_{3} h_{3}^{3}}{12\left(1-\nu_{3}^{2}\right)}\right)(1+Y)
\end{aligned}
$$

Similarly to previous paragraph, and assuming small loss factors $\eta_{1}$ and $\eta_{3}$, the asymptotic values of the equivalent loss factor of the three-layer $\left(\eta_{\mathrm{eq}}=\Im\left(D_{\mathrm{eq}}^{*}\right) / \Re\left(D_{\mathrm{eq}}^{*}\right)\right)$ can be derived:

$$
\begin{aligned}
\eta_{\mathrm{eq}, \mathrm{HF}} \approx \frac{\eta_{1} \frac{E_{1} h_{1}^{3}}{1-\nu_{1}^{2}}+\eta_{3} \frac{E_{3} h_{3}^{3}}{1-\nu_{3}^{2}}}{\frac{E_{1} h_{1}^{3}}{1-\nu_{1}^{2}}+\frac{E_{3} h_{3}^{3}}{1-\nu_{3}^{2}}} \\
\left.\eta_{\mathrm{eq}, \mathrm{LF}} \approx \eta_{\mathrm{eq}, \mathrm{HF}}+\frac{\Im\left(Y^{*}\right)}{1+\Re\left(Y^{*}\right)}\right)
\end{aligned}
$$

Finally, note that the maximum value of $\eta_{\text {eq }}$ is obtained for an optimal value of the shear parameter $X$ depending only on the geometrical parameter $Y$ and the loss factor of the layer 2. This optimum shearing of the dissipative core layer occurs under model of RKU for [41]:

$$
X_{\mathrm{opt}}=\frac{1}{\sqrt{(1+Y)\left(1+\eta_{2}^{2}\right)}}
$$

\section{B The Lamb waves model}

The Lamb waves model, originally due to H. Lamb, 1917 [H. Lamb, On waves in an elastic plate, Proc. Roy. Soc. (London), Ser. A, 93:1124, 1917], is well described in a text book by Viktorov [22]. The Lamb waves model is used in this paper to model 
a three-layered plate to compute the dispersion relationships of the plate. Each layer is modeled individually by means of the Lamb waves theory for infinite plates. The three-layered plate is considered to be in vacuum. The coordinate system $(x, z)$ and thicknesses of each layer, $h_{i}, i=1 \ldots 3$, are shown in Figure B.1.

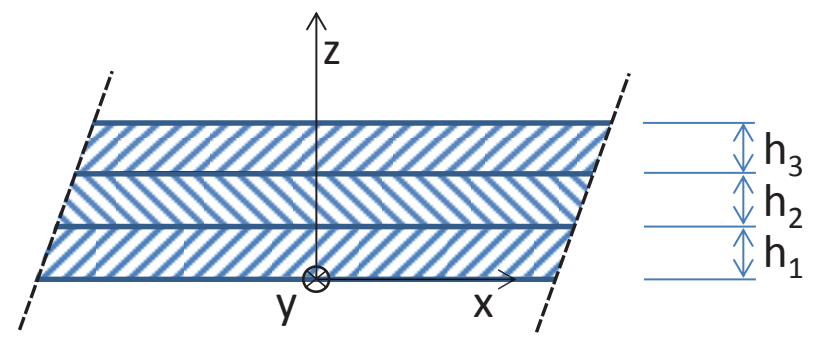

Figure B.1. Two-dimensional multilayer plate configuration.

The Lamb waves are described by two potentials $\phi_{i}$ and $\psi_{i}$ for the longitudinal and transversal wave components, respectively, for each layer $i, i=1 \ldots 3$, satisfying the wave equations

$$
\frac{\partial^{2} \phi_{i}}{\partial x^{2}}+\frac{\partial^{2} \phi_{i}}{\partial z^{2}}+k_{L, i}^{2} \phi=0 \quad \text { and } \quad \frac{\partial^{2} \psi_{i}}{\partial x^{2}}+\frac{\partial^{2} \psi_{i}}{\partial z^{2}}+k_{T, i}^{2} \psi=0
$$

where $k_{\mathrm{L}, \mathrm{i}}=\omega / c_{L, i}$ and $k_{\mathrm{T}, \mathrm{i}}=\omega / c_{\mathrm{T}, i}$ are the longitudinal and transverse wave numbers of each plate $i$, respectively, and where $c_{\mathrm{L}, i}=\sqrt{\left(\lambda_{i}+2 \mu_{i}\right) / \rho_{i}}$ and $c_{\mathrm{T}, i}=$ $\sqrt{\mu_{i} / \rho_{i}}$ are the longitudinal and transverse propagation speeds of each plate $i$, respectively. Furthermore, $\lambda_{i}$ and $\mu_{i}$ are the Lamé parameters of layer $i$, and $\rho_{i}$ is the density of layer $i$, and $\omega$ is the angular frequency. The displacement field of the particle displacements $u_{x, i}$ and $u_{z, i}$ in $x$ and $z$ directions, respectively, may be represented in terms of the potentials as

$$
u_{x, i}=\frac{\partial \phi_{i}}{\partial x}-\frac{\partial \psi_{i}}{\partial z} \quad \text { and } \quad u_{z, i}=\frac{\partial \phi_{i}}{\partial z}+\frac{\partial \psi_{i}}{\partial x}
$$

The mechanical stresses in each layer $i$ can be represented in terms of the displacements as

$$
\begin{array}{r}
\sigma_{x x, i}=\rho_{i}\left\{\left(c_{\mathrm{L}, i}^{2}-2 c_{\mathrm{T}, i}^{2}\right) \frac{\partial u_{z, i}}{\partial z}+c_{\mathrm{L}, i}^{2} \frac{\partial u_{x, i}}{\partial x}\right\} \\
\sigma_{z z, i}=\rho_{i}\left\{\left(c_{\mathrm{L}, i}^{2}-2 c_{\mathrm{T}, i}^{2}\right) \frac{\partial u_{x, i}}{\partial x}+c_{\mathrm{L}, i}^{2} \frac{\partial u_{z, i}}{\partial z}\right\} \\
\sigma_{z x, i}=\rho_{i} c_{\mathrm{T}, i}^{2}\left(\frac{\partial u_{x, i}}{\partial z}+\frac{\partial u_{z, i}}{\partial x}\right)
\end{array}
$$

Solutions of the wave equation B.1 for each layer are seeked using the following potentials 


$$
\begin{aligned}
& \phi_{i}=\left(A_{i} \mathrm{e}^{-\left(k^{2}-\omega^{2} / c_{\mathrm{L}, i}^{2}\right) z}+B_{i} \mathrm{e}^{\left(k^{2}-\omega^{2} / c_{\mathrm{L}, i}^{2}\right) z}\right) \mathrm{e}^{\mathrm{j}(\omega t-k x)} \\
& \psi_{i}=\left(C_{i} \mathrm{e}^{-\left(k^{2}-\omega^{2} / c_{\mathrm{T}, i}^{2}\right) z}+D_{i} \mathrm{e}^{\left(k^{2}-\omega^{2} / c_{\mathrm{T}, i}^{2}\right) z}\right) \mathrm{e}^{\mathrm{j}(\omega t-k x)}
\end{aligned}
$$

where $k$ is the wavenumber of the mechanical wave running through the multilayer plate and $A_{i}, B_{i}, C_{i}, D_{i}, i=1 \ldots 3$ are the unknown amplitudes of the wave components. A system of equations can be set up using the following boundary conditions.

Continuity of displacements at the interfaces of the layers:

$$
\begin{aligned}
& \left.u_{x, 1}\right|_{z=h_{1}}=\left.u_{z, 2}\right|_{z=h_{1}} \quad \text { and }\left.\quad u_{x, 2}\right|_{z=h_{1}+h_{2}}=\left.u_{z, 3}\right|_{z=h_{1}+h_{2}} \\
& \left.u_{z, 1}\right|_{z=h_{1}}=\left.u_{z, 2}\right|_{z=h_{1}} \quad \text { and }\left.\quad u_{z, 2}\right|_{z=h_{1}+h_{2}}=\left.u_{z, 3}\right|_{z=h_{1}+h_{2}}
\end{aligned}
$$

Continuity of stresses at the interfaces of the layers:

$$
\begin{aligned}
\left.\sigma_{z z, 1}\right|_{z=h_{1}}=\left.\sigma_{z z, 2}\right|_{z=h_{1}} & \text { and } & \left.\sigma_{z z, 2}\right|_{z=h_{1}+h_{2}}=\left.\sigma_{z z, 3}\right|_{z=h_{1}+h_{2}} \\
\left.\sigma_{z x, 1}\right|_{z=h_{1}}=\left.\sigma_{z z, 2}\right|_{z=h_{1}} & \text { and } & \left.\sigma_{z x, 2}\right|_{z=h_{1}+h_{2}}=\left.\sigma_{z z, 3}\right|_{z=h_{1}+h_{2}}
\end{aligned}
$$

Stress in normal direction is zero at the outer surfaces of the multilayer (the multilayer plate is assumed to be in vacuum):

$$
\begin{array}{rrrl}
\left.\sigma_{z z, 1}\right|_{z=0}=0 & \text { and } & \left.\sigma_{z z, 3}\right|_{z=h_{1}+h_{2}+h_{3}}=0 \\
\left.\sigma_{z x, 1}\right|_{z=0}=0 & \text { and } & \left.\sigma_{z x, 3}\right|_{z=h_{1}+h_{2}+h_{3}}=0
\end{array}
$$

For a three-layered plate a system of $3 \times 4=12$ equations and 12 unknowns amplitudes can be set up. In order to find non-trivial solutions the determinant of the resulting matrix is set to zero. The search for vanishing determinant is done numerically, for each angular frequency $\omega$, resulting in a frequency dependent dispersion relation between $k$ and $\omega$.

\section{Acknowledgments}

This work was performed within the framework of the Labex CeLyA of Université de Lyon, operated by the French National Research Agency (ANR-10-LABX0060/ ANR-11-IDEX-0007), It was partly funded by INSA-Lyon (BQR VIVARIUM project). The authors would like to thank Céline Sandier (engineer at LVA), Youssef Gerges (postdoctoral fellow at LVA), and master's students Valentin Henry, 
Eddy Fasana and Nhu Hai Nguyen for their precious help during measurements. We also express our gratitude to Jean-Louis Guyader for fruitful discussions.

\section{References}

[1] E. Nilsson, A. C. Nilsson, Prediction and measurement of some dynamic properties of sandwich structures with honeycomb and foam cores, Journal of Sound and Vibration 251 (3) (2002) $409-430$.

URL http://www.sciencedirect.com/science/article/pii/ S0022460X01940076

[2] D. Backström, A. C. Nilsson, Modelling the vibration of sandwich beams using frequency-dependent parameters, Journal of Sound and Vibration 300 (3-5) (2007) 589-611.

URL http://www.sciencedirect.com/science/article/pii/ S0022460X06007115

[3] J. Berthaut, M. Ichchou, L. Jezequel, K-space identification of apparent structural behaviour, Journal of Sound and Vibration 280 (3) (2005) 1125 - 1131.

URL http://www.sciencedirect.com/science/article/pii/ S0022460X04002925

[4] R. Cherif, J.-D. Chazot, N. Atalla, Damping loss factor estimation of two-dimensional orthotropic structures from a displacement field measurement, Journal of Sound and Vibration 356 (2015) $61-71$.

URL http://www.sciencedirect.com/science/article/pii/ S0022460X1500543X

[5] J. Cuenca, F. Gautier, L. Simon, The image source method for calculating the vibrations of simply supported convex polygonal plates, Journal of Sound and Vibration 322 (4) (2009) 1048 - 1069.

URL http://www.sciencedirect.com/science/article/pii/ S0022460X08009334

[6] J. Cuenca, F. Gautier, L. Simon, Measurement of complex bending stiffness of a flat panel covered with a viscoelastic layer using the image source method, in: Euronoise 2009, Edinburgh, 2009.

[7] A. Berry, O. Robin, F. Pierron, Identification of dynamic loading on a bending plate using the virtual fields method, Journal of Sound and Vibration 333 (26) (2014) 7151 -7164 .

URL http://www.sciencedirect.com/science/article/pii/ S0022460X1400710X

[8] A. Berry, O. Robin, P. O’Donoughue, Identifying dynamic constitutive parameters of bending plates using the virtual fields method, in: International Digital Imaging Correlation Society: Proceedings of the First Annual Conference, 2016, Springer International Publishing, 2017, pp. 5-8. 
URL

https://link.springer.com/chapter/10.1007\%

2F978-3-319-51439-0_2

[9] D. J. Ewins, Modal Testing: Theory, Practice and Application, 2nd Edition, Wiley, Research studies press, UK, 2000.

[10] K. Ege, X. Boutillon, B. David, High-resolution modal analysis, Journal of Sound and Vibration 325 (4-5) (2009) 852-869.

URL http://www.sciencedirect.com/science/article/pii/ s0022460X09003447

[11] Q. Leclère, F. Ablitzer, C. Pézerat, Practical implementation of the corrected force analysis technique to identify the structural parameter and load distributions, Journal of Sound and Vibration 351 (Supplement C) (2015) 106 - 118.

URL http://www.sciencedirect.com/science/article/pii/ S0022460X15003570

[12] N. B. Roozen, Q. Leclere, K. Ege, Y. Gerges, Estimation of plate material properties by means of a complex wavenumber fit using hankel's functions and the image source method, Journal of Sound and Vibration 390 (2017) 257-271.

URL http://www.sciencedirect.com/science/article/pii/ S0022460X16307118

[13] E. Carrera, An assessment of mixed and classical theories on global and local response of multilayered orthotropic plates, Composite Structures 50 (2) (2000) $183-198$.

URL http://www.sciencedirect.com/science/article/pii/ S0263822300000994

[14] P. Shorter, Wave propagation and damping in linear viscoelastic laminates, Journal of the Acoustical Society of America 115 (5) (2004) 1917-1925.

[15] E. Manconi, E. and B. R. Mace, Estimation of the loss factor of viscoelastic laminated panels from finite element analysis, Journal of Sound and Vibration 329 (19) (2010) $3928-3939$.

URL http://www.sciencedirect.com/science/article/pii/ S0022460X1000266X

[16] S. Ghinet and N. Atalla, Modeling thick composite laminate and sandwich structures with linear viscoelastic damping, Computers \& Structures 89 (15) (2011) $1547-1561$. URL http://www.sciencedirect.com/science/article/pii/ S0045794911001295

[17] J.-L. Guyader, C. Lesueur, Acoustic transmission through orthotropic multilayered plates, part i: plate vibration modes, Journal of Sound and Vibration 58 (1) (1978) $51-58$.

[18] J.-L. Guyader, C. Cacciolati, Viscoelastic properties of single layer plate material equivalent to multi-layer composite plate, in: Proceedings of Inter-Noise, Istanbul, Turkey, 2007.

[19] D. Ross, E. E. Ungar, E. Kerwin, Damping of plate flexural vibrations by means of viscoelastic laminae, In: Structural damping (J.E. Ruzicka, ed) 3 (1959) 44-87. 
[20] E. M. Kerwin, Damping of flexural waves by a constrained viscoelastic layer, Journal of the Acoustical Society of America 31 (7) (1959) 952-962.

URL : / /WOS:A1959WK54400013

[21] E. E. Ungar, E. M. Kerwin, Loss factors of viscoelastic systems in terms of energy concepts, Journal of the Acoustical Society of America 34 (7) (1962) 954-957.

URL : / /WOS:A19622950B00023

[22] I. A. Viktorov, Rayleigh and Lamb Waves, Plenum, New York, 1970.

[23] K. Ege, V. Henry, Q. Leclère, R. G. Rinaldi, C. Sandier, Vibrational behavior of multilayer plates in broad-band frequency range: comparisons between experimental and theoretical estimations, in: Proceedings of Inter-Noise, San Francisco, USA, 2015.

[24] J. P. H. Ahid D. Nashif, David I. G. Jones, Vibration Damping, John Wiley \& Sons, 1985.

[25] D. N. Alleyne, P. Cawley, The interaction of lamb waves with defects, Ieee Transactions on Ultrasonics Ferroelectrics and Frequency Control 39 (3) (1992) 381397.

URL : / /WOS :A1992JA53600010

[26] Z. Q. Su, L. Ye, Y. Lu, Guided lamb waves for identification of damage in composite structures: A review, Journal of Sound and Vibration 295 (3-5) (2006) 753-780.

URL : / /WOS: 000238844300018

[27] R. Roy, T. Kailath, Esprit - estimation of signal parameters via rotational invariance techniques, IEEE Transactions on Acoustics Speech and Signal Processing 37 (7) (1989) 984-995.

[28] R. Badeau, B. David, R. Richard, A new perturbation analysis for signal enumeration in rotational invariance techniques, IEEE Transactions on Signal Processing 54 (2) (2006) 450-458.

[29] J. Laroche, The use of the matrix pencil method for the spectrum analysis of musical signals, Journal of the Acoustical Society of America 94 (4) (1993) 1958-1965.

[30] K. Ege, X. Boutillon, M. Rébillat, Vibroacoustics of the piano soundboard: (non)linearity and modal properties in the low- and mid-frequency ranges, Journal of Sound and Vibration 332 (5) (2013) 1288-1305.

URL http://www.sciencedirect.com/science/article/pii/ S0022460X12007936

[31] K. Ege and T. Boncompagne and B. Laulagnet and J.-L. Guyader, Experimental estimations of viscoelastic properties of multilayer damped plates in broad-band frequency range, in: Proceedings of Inter-Noise, Vol. 2, New-York, USA, 2012, pp. $1575-1583$.

[32] H. D. Hwang and K. Ege and L. Maxit and N. Totaro and J.-L. Guyader, A methodology for including the effect of a damping treatment in the mid-frequency domain using SmEdA method, in: Proceedings of 20th International Congress on Sound and Vibration 2013, ICSV 2013, Vol. 1, Bangkok, Thailand, 2013, pp. 636644. 
[33] H. D. Hwang, L. Maxit, K. Ege, Y. Gerges, J.-L. Guyader, SmEdA vibro-acoustic modelling in the mid-frequency range including the effect of dissipative treatments, Journal of Sound and Vibration 393 (2017) 187 - 215.

URL http://www.sciencedirect.com/science/article/pii/ S0022460X17300470

[34] C. Pézerat, J.-L. Guyader, Force analysis technique: Reconstruction of force distribution on plates, Acustica united with Acta Acustica 86 (2000) 322-332.

[35] Q. Leclère, C. Pézerat, Vibration source identification using corrected finite difference schemes, Journal of Sound and Vibration 331 (6) (2012) 1366 - 1377.

URL http://www.sciencedirect.com/science/article/pii/ S0022460X11008807

[36] F. Ablitzer, C. Pézerat, J.-M. Génevaux, J. Bégué, Identification of stiffness and damping properties of plates by using the local equation of motion, Journal of Sound and Vibration 333 (9) (2014) 2454 - 2468.

URL http://www.sciencedirect.com/science/article/pii/ S0022460X13010535

[37] Q. Leclère, F. Ablitzer, C. Pézerat, Identification of loads of thin structures with the corrected Force Analysis technique: An alternative to spatial filtering regularization, in: Proceedings of ISMA 2014, Leuven, Belgique, 2014.

URL http: //hal.archives-ouvertes.fr/hal-01057679

[38] J.-F. Blachot, L. Shazeau, Y. Cavaille, Rheological behavior of cellulose/monohydrate of n-methylmorpholine n-oxide solutions. part 2. glass transition domain, Polymer 43 (3) (2002) $881-889$.

URL http://www.sciencedirect.com/science/article/pii/ S0032386101006310

[39] M. Gallo and R. G. Rinaldi.and L. Chazeau and J.-M. Chenal and F. Ganachaud and Q. Leclère and K. Ege and N. Totaro, Versatile hybrid sandwich composite combining large stiffness and high damping: spatial patterning of the viscoelastic core layer, in: Proceedings of Acoustics'2017, Vol. 141:5, Boston, USA, 2017, pp. 3643-3643. URL https://doi.org/10.1121/1.4987867

[40] N. B. Roozen, L. Labelle, Q. Leclère, K. Ege, S. Alvarado, Non-contact experimental assessment of apparent dynamic stiffness of constrained-layer damping sandwich plates in a broad frequency range using a Nd:YAG pump laser and a laser Doppler vibrometer, Journal of Sound and Vibration 395 (2017) 90 - 101.

URL http://www.sciencedirect.com/science/article/pii/ S0022460X17301050

[41] I. Vér, L. Beranek, Noise and Vibration Control Engineering, Second Edition, Chapter 14 - Structural Damping - E. Ungar and J.A.Zapfe, John Wiley \& Sons, 2006. 


\section{List of Figures}

1 Comparison of the three models for the thin and soft flexural polymer interlayer "SOFT" plate - Equivalent rigidity $D_{\text {eq }}(f)$ (top) ; Equivalent loss factor $\eta_{\mathrm{eq}}$ (bottom). Low- and high-frequency asymptotic values are added in horizontal dashed lines, and maximum damping frequency is added in vertical dashed lines for both RKU model and Guyader's model.

2 Comparison of the three models for the steel-polymer-steel "SPS" plate - Equivalent rigidity $D_{\text {eq }}(f)$ at top and middle (zoom in high-frequency) ; Equivalent loss factor $\eta_{\mathrm{eq}}$ at bottom. Low- and high-frequency asymptotic values are added in horizontal dashed lines, and maximum damping frequency is added in vertical dashed lines for both RKU model and Guyader's model.

3 (a) Schematic illustration of the three-layered sandwich plate under study. (b) Optical microscope observation of the through-thickness cross sections of real samples: left - polymer core layer; right SPS sandwich composite.

4 Typical frequency responses "Acceleration/Force" (Fourier spectrum) for the SPS plate, obtained by impact-hammer excitation (see section 3.2.1 for the experimental protocol).

5 Comparisons of six experimental (left figure) and numerical (right figure) mode shapes of the SPS plate in the low-frequency domain (numerical mode shapes are extracted on the experimental mesh). For each mode, the MAC value and identified equivalent Young's modulus (by matching FEM estimation to measured modal frequency) are given in caption.

6 Block diagram of the high-resolution modal analysis method (see Ege et al. [10] for details).

7 Typical bank-filtering analysis of an impulse response of the SPS plate between 1060 and $1180 \mathrm{~Hz}$. (a) —: Fourier spectrum of the impulse response; - - -: amplitude response of a narrow-band filter. - marks: estimated modal amplitudes and modal frequencies. (b) - marks: measured loss factors $\eta_{k}$ for the four real modes (the number of complex exponentials $K=8 \quad(=2 \times 4)$ is estimated with ESTER criterion). (c) • marks: Energy of each components. 
8 Measurement set-up for the wave based characterization methodologies (CFAT and Hankel). (a) Laser vibrometer and plate. (b) Plate hanging on thin wires, excited by mini shaker. The black line on the plate indicates the measurement area.

9 Normalized velocity fields measured at 2(left), 4(center) and 8 (right) $\mathrm{kHz}$. The scanning area is $11 \times 11.6 \mathrm{~cm}$.

10 Real part of $D /(\rho h)$ estimated using CFAT with different scale factors $\Delta / \delta$.

11 Rough estimation of $k_{\mathrm{N}}$ and resulting selection of the scale factor used for CFAT. (a) 2DFFT-based estimation of $k_{\mathrm{N}}$ (red dots) and fitted regression (black line). (b) Selected scale factor (top), and ratio between $\lambda_{\mathrm{N}}$ and $\Delta$ (bottom).

12 Equivalent Young's modulus $E_{\mathrm{eq}}$ function of the frequency for the steel-polymer-steel (SPS) plate.

13 Equivalent loss factor $\eta_{\mathrm{eq}}$ function of the frequency for the steel-polymer-steel (SPS) plate.

14 GABO Explexor DMA apparatus with tensile grips (with zoom-in from left to right).

15 Experimental Young's modulus and loss factor of the polymer core layer as a function of temperature.

16 Measured and predicted Young's modulus of the polymer core versus temperature for varying frequencies. The predictions are obtained based on the time-temperature superposition principle.

17 Predicted Young's modulus and loss factor of the polymer core versus frequency at ambient temperature $(296 \mathrm{~K})$.

A.1 Three-layered constrained layer plate.

B.1 Two-dimensional multilayer plate configuration. 


\section{List of Tables}

1 Dimensions and material properties of the individual layers for the two constrained-layer damping sandwich plates chosen for analytical comparison.

2 Relevant analytical results obtained for SOFT and SPS plates. 